\title{
The anomalous Mössbauer fraction of ferritin and polysaccharide iron complex (PIC)
}

\author{
M-E.Y. Mohie-Eldin ${ }^{\mathrm{a}, 1}$, R.B. Frankel ${ }^{\mathrm{b}}$, L. Gunther ${ }^{\mathrm{a}}$ and G.C. Papaefthymiou ${ }^{\mathrm{c}}$ \\ ${ }^{a}$ Department of Physics and Astronomy, Tufts University, Medford, \\ MA 02155, USA \\ ${ }^{\mathrm{b}}$ Physics Department, California Polytechnic State University, \\ San Luis Obispo, CA 93407, USA \\ ${ }^{c}$ Francis Bitter National Magnet Laboratory, Massachusetts Institute of Technology, \\ Cambridge, MA 02139, USA
}

Mössbauer studies of the ubiquitous protein molecule ferritin and its synthetic "biomimic" polysaccharide iron complex (PIC) exhibit an anomaly in the Mössbauer spectrum wherein the recoil free fraction or $f$-factor has a sharp drop with respect to temperature as the temperature rises above $30 \mathrm{~K}$ for mammalian ferritin and $60 \mathrm{~K}$ for PIC. The anomaly coincides with the disappearance of hyperfine splitting, which is due to superparamagnetic relaxation above the blocking temperature. Different absorbers were used to experimentally investigate the effect of absorber thickness on the Mössbauer spectrum. The anomaly persists for thin absorbers. Also, spectra treated with FFT procedures to eliminate the thickness effect still exhibit this anomaly. Motion of the core with respect to the protein shell was also eliminated as a possible source for this phenomenon, by comparing the Debye temperature obtained from the temperature dependence of the $f$-factor and the isomer shift. A comparison of the magnetic anisotropy constants from magnetization studies with those obtained by relating the hyperfine field $H$ of the Mössbauer spectra to the fluctuations of the magnetization imply that the ferritin and PIC molecules possess magnetic anisotropy energy which may not be strictly uniaxial. This, we believe, may be intimately connected with the mechanism causing the $f$-factor anomaly.

\section{Ferritin}

Ferritin is an ubiquitous protein widespread among plants, animals, and in several bacteria, which is designed to store and maintain iron in an available, nontoxic form [1-3]. In all forms, the molecule consists of a hydrous ferric oxide core

1 This work was carried out in partial fulfilment of the requirements of the PhD degree at Tufts University. 
sequestered in a roughly spheroidal, $120 \AA$ diameter protein shell. The protein shell, called "apoferritin", is composed of 24 nearly identical sub-units of molecular weight $\sim 20000$ daltons, which are arranged to isolate the iron containing core from the cellular environment. Six hydrophilic and eight hydrophobic channels provide access to the protein interior, persumably for electrons, protons, and iron ions, as well as other small ions.

The mammalian ferritin iron core is a hydrous ferric oxide phosphate with nominal formula $(\mathrm{FeOOH})_{8}\left(\mathrm{FeO} \cdot \mathrm{H}_{2} \mathrm{PO}_{4}\right)$, a structure similar to the polycrystalline mineral ferrihydrite. It contains $\mathrm{Fe}^{3+}$ ions octahedrally coordinated to oxygen, i.e. six-fold oxygen coordination, in a crystalline array, and oxygens are hexagonally close packed [4]. Phosphate occurs in disordered regions of the core, possibly at the chain ends of the iron polymer and/or at the junction of crystallites with each other or with the protein surface. The core can store up to a maximum of 4500 iron atoms (ions) [5]. When saturated with iron, the core has a diameter of about $80 \AA$ [5] which is the inner cavity dimension of the protein shell. The entire ferritin molecule has a molecular weight of about 700000 daltons. The function of the ferritin molecule has been shown [6] to store iron in an available non-toxic form in the various biological organisms in which it is found. However, despite recent advances [7-11] the exact structure of the iron mineral core, as well as a definite method by which the ferritin molecule fulfills its function, are still elusive.

\section{Lamb-Mössbauer $f$-factor (Mössbauer fraction) anomaly in ferritin}

Mössbauer spectra of the ferritin molecule have been shown [12] to consist of a magnetic hyperfine six line spectrum at low temperatures, which tends to an electric quadrupole doublet at higher temperatures. At the Francis Bitter National Magnet Laboratory at MIT, the Lamb-Mössbauer $f$-factor was obtained for a wide range of temperatures: $T=4.2-300 \mathrm{~K}$. Fig. 1 shows the $f$-factor normalized to $4.2 \mathrm{~K}$ data for the naturally occurring mammalian ferric ferritin. A description of this sample has already been given elsewhere [13]. All published work on ferritin known to us treated the $f$-factor or temperature dependence for $T \geqslant 80 \mathrm{~K}$, where only paramagnetic (i.e. quadrupole split) spectra were observed. One obtains a smooth dependence with the spectral area $(f$-factor) saturating at $\sim 100 \mathrm{~K}$. The reason for this is that according to the Debye theory [14] the $f$-factor is expected to approach a plateau at low temperatures. For ferritin, the plateau was assumed to have been reached at $T=80 \mathrm{~K}$. Thus, it was assumed that $f(T=80 \mathrm{~K}) \approx$ $f(T=0 \mathrm{~K})$. When measurements were extended to lower temperatures, hyperfine structure appears, as expected for such superparamagnetic molecules. In addition, however, we found that the $f$-factor shows a further apparent anomalous increase with decreasing temperatures around the blocking temperature of mam- 


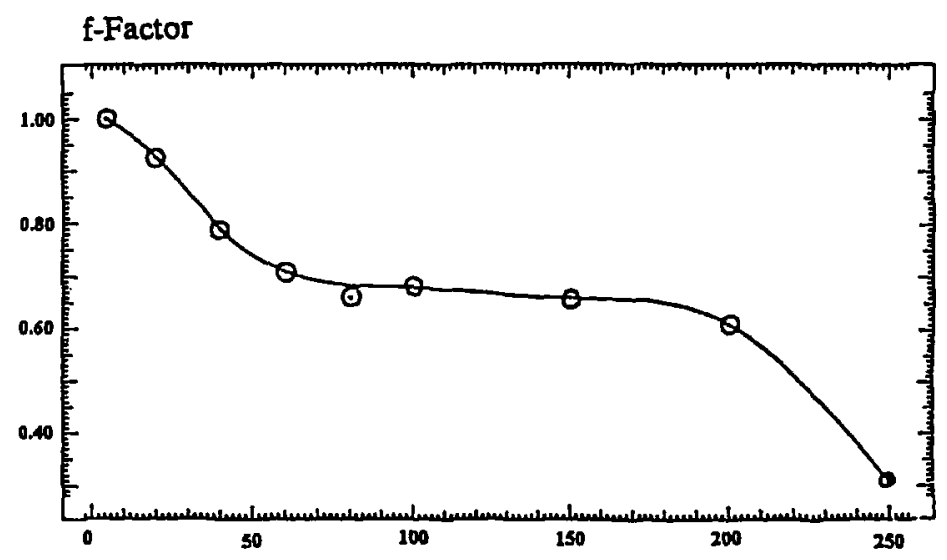

Temperature (K)

Fig. 1. Total Mössbauer area of ferric ferritin normalized to the $4.2 \mathrm{~K}$ data.

malian ferritin $T_{\mathrm{B}}=37 \mathrm{~K}$, which is the temperature at which the spectral area of the hyperfine sextet and the quadrupole doublet become equal.

\section{Polysaccharide iron complex (PIC)}

PIC is a synthetic complex of ferric iron and carbohydrate marketed under the name Niferex as an oral hematinic by Central Pharmaceuticals, Inc. (Seymour, Indiana). It is reported to be effective in the treatment of iron deficiency anemia [15]. PIC is reported [16-19] to be spheroidal in shape with an $48 \%$ iron content, coated with carbohydrate material, and with an average core diameter less than the maximum core diameter $(80 \AA)$ of mammalian ferritin. PIC is synthesized on an industrial scale by Central Pharmaceuticals, Inc. essentially by a patented procedure [18] which is described in detail elsewhere [16]. Berg [16] has presented evidence from Mössbauer spectroscopy and X-ray diffraction, which are fast (response time $\leqslant 10^{-7}$ ) short-range probing techniques, that the iron core in PIC is similar to ferrihydrite and ferritin. This has been confirmed by [20] the use of long-range order probing techniques, such as magnetization measurements to investigate and compare the magnetic properties of PIC and ferritin. These properties indicated that PIC has a greater blocking temperature $T_{B}$ than that of mammalian ferritin. Based on the above, as well as its ease of handling and synthesis and its stability over a wider range of temperatures, we used the synthetic polysaccharide iron complex molecule as a useful "biomimic" model with which to compare the anomaly occurring in ferritin, and more importantly, to investigate whether the simultaneous appearance of hyperfine structure near the blocking temperature with the anomalous increase of the $f$-factor is coincidental or related. 


\section{Experiment}

Mössbauer measurements were made at the Francis Bitter National Magnet Laboratory at MIT PIC was used to produce five Mössbauer absorbers containing $10 \mathrm{mg} \mathrm{PIC} / \mathrm{cm}^{2}, 20 \mathrm{mg} \mathrm{PIC} / \mathrm{cm}^{2}, 35 \mathrm{mg} \mathrm{PIC} / \mathrm{cm}^{2}, 50 \mathrm{mg} \mathrm{PIC} / \mathrm{cm}^{2}, 75 \mathrm{mg} \mathrm{PIC} /$ $\mathrm{cm}^{2}$, and $100 \mathrm{mg} \mathrm{PIC} / \mathrm{cm}^{2}$. Each absorber was mixed with boron nitride and pressed to uniform thickness. The absorbers were sealed and mounted into a super vary temp liquid helium cryostat which provided a controlled absorber temperature from $T=4.2$ to $295 \mathrm{~K}$.

The absorber was in an exchange gas environment, and the temperature was controlled by a feedback system. Our configuration was a conventional one, where the source was a $\sim 50 \mathrm{mCi}{ }^{57} \mathrm{Co}(\mathrm{Rh})$ (New England Nuclear) which was maintained at room temperature. The spectra were calibrated using the Mössbauer spectrum of an iron foil at room temperature.

Data was analyzed by least squares using a fitting routine on a VAX ( 5000 series) computer at the Francis Bitter National Magnet Laboratory. The program used for data analysis was originally written by Varret and Teillet [21]. The program could fit the observed data to a calculated broad distribution of magnetic hyperfine fields, as well as to a broad distribution of quadrupole splitting electric field gradients at the ${ }^{57} \mathrm{Fe}$ nucleus. We note that all individual lines in these procedures were assumed to have Lorentzian line shape.

For this program, for each absorber, the spectral line width was fixed at a value that gave the best fit for all temperatures. The total intensity, which is the sum of the individual line intensities, was allowed to vary and the isomer shift (IS) was also allowed to vary. For the sextet distributions, the quadrupole splitting (QS) was also allowed to vary. The output of this program is the determined values of the varied parameters, plus a probability distribution for the magnetic hyperfine or quadrupole splitting distributions which best fits the experimental data. This program calculated the area under the spectrum for the raw data and from the fit. The two were usually very close (to within $\leqslant 0.01 \%$ ). It also calculated the relative peak areas for each sextet and/or doublet component: $P_{\mathrm{s}}, P_{Q}$, respectively. Another simple program was used to calculate the raw area under the spectrum as well as the normalized area under the spectrum: $\sum_{i}^{513}\left[\left(N(\infty)-N_{i}(v)\right) / N(\infty)\right]$, where $N(v)$ is the number of counts (per channel) at velocity $v$ and $N(\infty)$ is the number of counts (per channel) at velocities far away from the resonant velocities, i.e. in the wings of the spectra. In addition, it determined individual line intensities from the raw data. The raw area under the spectrum obtained from this program was checked with that of the fitting program. However, the normalized area and line intensities were used in the absorber thickness effect analysis. Errors in the areas were computed from the raw data error in the number of counts per channel per spectrum, determined by a statistical routine in the program that formats the transferred raw data from the Mössbauer spectrometer. 


\section{Results}

\subsection{CONFIRMATION OF THE LAMB-MÖSSBAUER $f$-FACTOR ANOMALY IN PIC}

Based on the previously mentioned arguments indicating the similarity of PIC and ferritin $[16,20]$, we examined the possibility that the $f$-factor anomaly observed in mammalian ferritin might also be exhibited by the PIC molecule, by initially measuring the Mössbauer spectra of a $35 \mathrm{mg} \mathrm{PIC} / \mathrm{cm}^{2}$ absorber for $T=4.2 \mathrm{~K}$ to $T=295 \mathrm{~K}$. The area under the spectra was evaluated using the above mentioned programs. In fig. 2 , the normalized area to the $4.2 \mathrm{~K}$ data is plotted versus temperature. The anomalous $f$-factor below $100 \mathrm{~K}$ is evident and coincides with PIC's (higher) blocking temperature $T_{\mathrm{B}}=63 \mathrm{~K}$ just as observed in mammalian ferritin.

\subsubsection{Absorber thickness as a possible anomaly source}

In general, Mössbauer spectra are occasionally affected by an "absorber thickness" effect. This effect is due to the fact that the spectrum of $\gamma$-rays incident on a given nucleus in the interior of the absorber has been modified by the non-uniform absorption of $\gamma$-rays by the nuclei that are in front of the given nucleus. The absorption spectrum for a given spectral line is then not a Lorentzian. The question we first confronted was whether the thickness effect was responsible for the apparent anomaly. We used two approaches: first, we studied a series of samples having varied thickness and extrapolated our results down to zero thickness. Second, we used a deconvolution procedure to remove the thickness effect from our data.

In a Mössbauer effect (ME) experiment, the counting rate $N(v)$ of $\gamma$-rays transmitted through the absorber is measured as a function of the velocity $v$ of the source with respect to the absorber. At sufficiently high velocities no resonant absorption

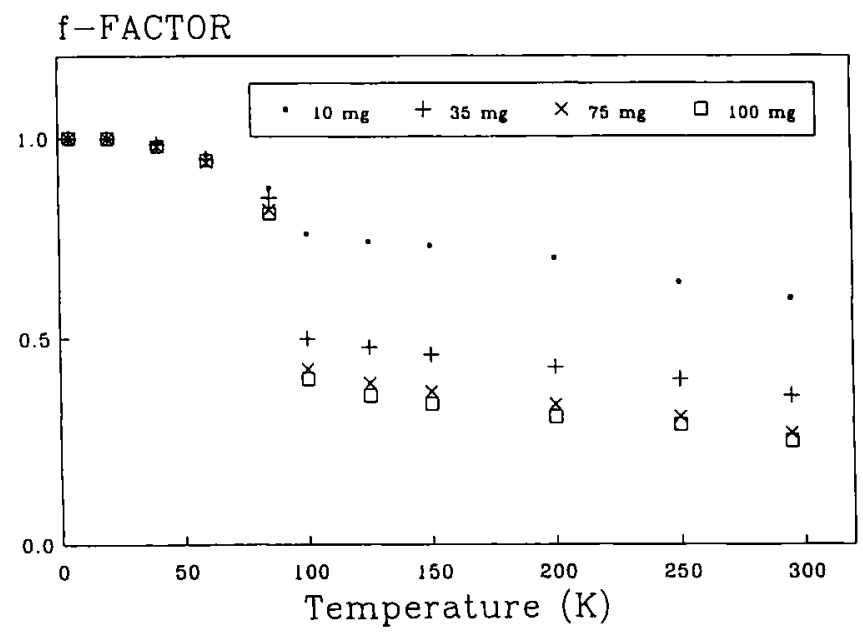

Fig. 2. $f$-factor for the $10,35,75$, and $100 \mathrm{mg} / \mathrm{cm}^{2} \mathrm{PIC}$ absorbers versus temperature. 
takes place, and if we let $V=E_{0}(1+v / c)$, where $E_{0}$ is the energy at which maximum absorption occurs, and $c$ is the speed of light, hence for large $V$,

$$
N(V) \rightarrow N(\infty)=N_{\mathrm{B}}+N_{\gamma},
$$

where $N_{\mathrm{B}}$ is the counting rate due to background radiation and $N_{\gamma}$ that of the $\gamma$-rays of the ME transition. At lower velocities $N(V)$ can be written as

$$
N(V)=N(\infty)-\epsilon(V) N_{\gamma},
$$

where $\epsilon(V)$ is the fraction of $N_{\gamma}$ which is absorbed recoil free at velocity $v$ (i.e. at energy $V$ ). This fraction can be expressed as

$$
\epsilon(V)=f_{\mathrm{s}} \int_{-\infty}^{\infty} S(E-V)\left\{1-\exp \left[-t_{\mathrm{a}} \sigma_{\mathrm{r}}(E)\right]\right\} \mathrm{d} E,
$$

where $f_{s}$ is the recoilless fraction, i.e. $f$-factor [14] of the source. $S(E-V)$ is the Lorentzian distribution of the gamma rays emitted by the source, where only gamma ray decay processes are considered,

$$
S(E-V)=\frac{1}{2 \pi} \frac{\Gamma_{\mathrm{n}}}{(E-V)^{2}+\left(\frac{1}{2} \Gamma_{\mathrm{n}}\right)^{2}} .
$$

$S(E-V)$ is normalized to unity, while $\Gamma_{\mathrm{n}}=$ the natural gamma line width $=$ $4.67 \times 10^{-9} \mathrm{eV}(\equiv 0.0973 \mathrm{~mm} / \mathrm{s}) . \sigma_{\mathrm{r}}(E)$ is the resonance absorption cross section given by

$$
\sigma_{\mathrm{r}}(E)=\frac{\Gamma_{\mathrm{n}}}{\Gamma_{\mathrm{a}}} \frac{\left(\frac{1}{2} \Gamma_{\mathrm{a}}\right)^{2}}{\left(E-E_{0}\right)^{2}+\left(\frac{1}{2} \Gamma_{\mathrm{a}}\right)^{2}},
$$

where $\Gamma_{\mathrm{a}}$ is the total width of the absorption line. The dimensionless quantity $t_{\mathrm{a}}$ is defined as

$$
t_{\mathrm{a}}=n_{\mathrm{a}} f_{\mathrm{a}} \sigma_{0},
$$

where $n_{\mathrm{a}}$ is the number of ME atoms $\left({ }^{57} \mathrm{Fe}\right)$ per unit area, $\sigma_{0}$ is the maximum resonant cross section $=\lambda^{2} / 2 \pi\left(2 I_{\mathrm{e}}+\frac{1}{2} I_{\mathrm{g}}+1\right)=2.363 \times 10^{-18} \mathrm{~cm}^{2}$, where $I_{\mathrm{e}}$ and $I_{\mathrm{g}}$ are the nuclear spins of the excited state and the ground state, respectively, and $\lambda=$ wavelength of the gamma radiation, while $f_{\mathrm{a}}$ is the recoilless fraction $(f$-factor) of the absorber.

The area $A$ of the Mössbauer spectrum can be estimated as

$$
A=\sum_{v} \frac{N(\infty)-N(V)}{N(\infty)-N_{\mathrm{B}}}=\sum_{m} \frac{N(\infty)-N(m)}{N(\infty)-N_{\mathrm{B}}},
$$

where $N(m)$ is the number of counts in channel $m$ (corresponding to the velocity $v$ ) of the multichannel analyzer. Hence using eqs. (1) and (2), one can write the following theoretical expression for $A$ : 


$$
A=\int_{-\infty}^{\infty} \epsilon(V) \mathrm{d} V=f_{\mathrm{s}} F_{\mathrm{l}}\left(t_{\mathrm{a}}\right) .
$$

$F_{1}\left(t_{\mathrm{a}}\right)$ is called the "absorption area function" and can be evaluated by inserting for $\epsilon(V)$ the integral of eq. (3). Changing the order of integrations, one obtains

$$
F_{1}\left(t_{\mathrm{a}}\right)=\int_{-\infty}^{\infty}\left\{1-\exp \left[-t_{\mathrm{a}} \sigma_{\mathrm{r}}(E)\right]\right\} \mathrm{d} E,
$$

where it is clear the absorption area is dependent on the absorber thickness, $t_{\mathrm{a}}$, the absorber recoilless fraction, $f_{\mathrm{a}}$, and its resonant cross-section, $\sigma_{\mathrm{r}}$.

We note that the practice in the literature of obtaining the $f$-factor $f_{\mathrm{a}}(T)$ of an absorber and its temperature dependence from the absorption area $A(T)$ under the Mössbauer spectra normalized to a particular temperature $T_{0}$, e.g. $A(T) / A\left(T_{0}\right)$, is based on the assumption that $A(T)$ is proportional to the recoilless fraction of the absorber $f_{\mathrm{a}}(T)$ and that the temperature dependence of $A(T)$ is the same as that of $f_{\mathrm{a}}(T)$. However, this is true only if the absorber thickness is small, and hence this would give in essence a relative recoilless fraction $f_{\mathrm{a}}(T)$.

At low temperatures, in the presence of unpaired electronic spin, the Mössbauer nucleus experiences a magnetic hyperfine field which splits the ground and excited nuclear spin states, thus providing six allowable transitions between the excited and ground states, each with a different relative transition probability and hence different resonant cross section. This splitting is reflected in the six lines of the Mössbauer spectrum and their areas. Still, the total resonant cross-section and the total area of the Mössbauer spectrum remain conserved.

It is important to note that such single line components, which reflect these six transitions with differing relative transition probabilities and resonant cross section, will show a difference in their saturation behavior (i.e. at infinite absorber thickness). This difference of saturation behavior will cause an apparent accentuation of the nominally weaker (hyperfine) components with increasing cross section, i.e. with increasing absorber thickness and/or with increasing recoilless fraction (decreasing temperature). This is illustrated by the calculated curves in fig. 3.7 on p. 71 of ref. [22], which shows the changes in the relative absorption area of two hyperfine lines caused by saturation effects with change in recoilless fraction and with change in absorber thickness. This demonstrates the overlapping effects of the absorber thickness and the recoilless fraction ( $f$-factor) on the spectral absorption area. Fig. 3 shows this well known $[14,22,23]$ dependence of the total resonance absorption area of a single line on the recoilless fraction, $f_{\mathrm{a}}$ and the absorber thickness, $t_{\mathrm{a}}$. From the behavior of the Mössbauer absorption area in fig. 3 it is evident that at low temperatures or large $f$-factor values, the effect of the thickness in increasing the spectral absorption area is strong, while at high temperatures or small values of the $f$-factor the effect of the thickness in increasing the spectral absorption is weak.

The thickness effect is essentially a reduction in the absorption of $\gamma$-rays from the linear dependence with respect to thickness that would otherwise obtain. The 


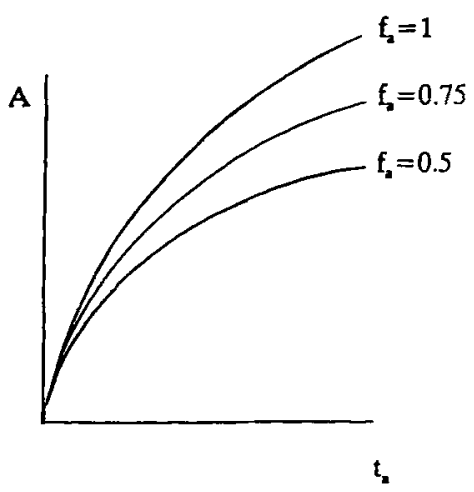

Fig. 3. Dependence of the Mössbauer spectrum resonance absorption area on $f_{\mathrm{a}}$ : recoilless absorption fraction, and $t_{\mathrm{a}}$ : absorption thickness.

reduction is greater the greater the degree of absorption otherwise present. As a consequence, for example, the thickness effect gives rise to a broadening of a given spectral line. Now, as the temperature increases, the Debye-Waller factor decreases. The lower the intrinsic absorption, the smaller will be the effect of the thickness effect. Hence, for a given temperature independent spectral line, the thickness effect will result in a slower drop in the observed absorption as a function of temperature. Thus, the thickness effect should weaken any anomaly of the sort we have observed, let alone produce it! However, in our case, the intrinsic spectrum is temperature dependent and in fact collapses from a sextet to a doublet as the temperature increases. The concentration of absorption over a smaller frequency range results in an increase in the thickness effect and hence a sharper drop of the total absorption with respect to increasing temperature. Thus it is that the thickness effect might account for the observed anomaly.

This conclusion may not be immediately apparent if one considers a more detailed inspection of the absorption area function (see eq. (9a)) and neglects to take the temperature dependence of the shape of $\sigma(E)$ into consideration. In order to understand better the above argument, let us begin by considering a spectrum that consists of a single line whose width is temperature independent. The integral in eq. (9a) can be solved analytically $[14,22,23]$,

$$
F_{1}\left(t_{\mathrm{a}}\right)=\pi \frac{1}{2} t_{\mathrm{a}} \exp \left(-\frac{1}{2} t_{\mathrm{a}}\right)\left[I_{0}\left(\frac{1}{2} t_{\mathrm{a}}\right)+I_{1}\left(\frac{1}{2} t_{\mathrm{a}}\right)\right]
$$

where $I_{n}(z)$ are the modified Bessel functions of order $n$. Calculating the ratio of Mössbauer absorption areas $A(T) / A\left(T_{0}\right)$, where $T_{0} \approx 0 \mathrm{~K}$, one finds that this ratio, the apparent "normalized" $f$-factor, increases with increasing absorber thickness. This implies the exact opposite of the above conclusion. In other words, in the case of a single Lorentzian, for large absorber thickness, the normalized $f$-factor is larger than for thin absorbers at constant temperature. This effect would negate the anomalous drop we observe in the $f(T)$ curves of our samples, 
and would automatically exclude the absorber thickness effect as a possible source of the anomaly.

However, let us consider a spectrum that is split into $n$ lines, each with width $\Gamma_{\mathrm{a}}$. The absorption cross section is given by

$$
\sigma(E)=\sum_{i=1}^{n} \frac{\beta_{i}\left(\frac{1}{2} \Gamma_{\mathrm{a}}\right)^{2}}{\left(E-E_{i}\right)^{2}+\left(\frac{1}{2} \Gamma_{\mathrm{a}}\right)^{2}},
$$

where $n=2$ or 6 in the case of quadrupole or magnetic hyperfine splitting respectively, $E_{i}$ is the energy of the $i$ th absorption line, and $\beta_{i}\left(\sum_{i=1}^{n} \beta_{i}=1\right)$ represents its partial strength. For simplicity, let us assume that the lines do not overlap. Each line may then be treated separately $[14,22,23]$ provided one allocates the absorber an effective reduced thickness $t_{\mathrm{a} i}=\beta_{i} t_{\mathrm{a}}$ for each line. Hence the absorption area function for the $i$ th line becomes:

$$
F_{1}\left(\beta_{i} t_{\mathrm{a}}\right)=\pi\left(\frac{1}{2} \beta_{i} t_{\mathrm{a}}\right) \exp \left(-\frac{1}{2} \beta_{i} t_{\mathrm{a}}\right)\left[I_{0}\left(\frac{1}{2} \beta_{i} t_{\mathrm{a}}\right)+I_{1}\left(\frac{1}{2} \beta_{i} t_{\mathrm{a}}\right)\right] .
$$

The value of $F_{1}\left(t_{\mathrm{a} i}\right)$ for each line increases since less saturation occurs [14,22,23]. Considering the Mössbauer spectra of our sample, which consist of a wide six-line hyperfine split spectrum with $\left(V_{1}-V_{6} \approx 15 \mathrm{~mm} / \mathrm{s}\right)$ at low temperatures, that tends to a narrow two-line quadrupole split spectrum with $\left(V_{1}-V_{2} \approx 0.6 \mathrm{~mm} / \mathrm{s}\right)$ at high temperatures, we find that the ratio of Mössbauer absorption areas for such spectra take the form

$$
\frac{A(T)}{A\left(T_{0}\right)}=\frac{\sum_{i=1}^{n} A_{i}(T)}{\sum_{i=1}^{6} A_{i}\left(T_{0}\right)} .
$$

Assuming $t_{\mathrm{a}}=t f_{\mathrm{a}}$ (see eq. (6)) and using eq. (9d) to calculate such a ratio, we get $\left[A(T) / A\left(T_{0}\right)\right]_{\ell \rightarrow 0}=f_{\mathrm{a}}(T) / f_{\mathrm{a}}\left(T_{0}\right)$ and

$$
\left[\frac{A(T)}{A\left(T_{0}\right)}\right]_{t \gg 1}=\left(\frac{f_{\mathrm{a}}(T)}{f_{\mathrm{a}}\left(T_{0}\right)}\right)^{1 / 2} \frac{\sum_{i=1}^{n} \sqrt{\beta_{i}}}{\sum_{i=1}^{6} \sqrt{\beta_{i}}}
$$

Hence we have

$$
\frac{\left[A(T) / A\left(T_{0}\right)\right]_{t \gg 1}}{\left[A(T) / A\left(T_{0}\right)\right]_{t \rightarrow 0}}=\left(\frac{f_{\mathrm{a}}\left(T_{0}\right)}{f_{\mathrm{a}}(T)}\right)^{1 / 2} \frac{\sum_{i=1}^{n} \sqrt{\beta_{i}(T)}}{\sum_{i=1}^{6} \sqrt{\beta_{i}\left(T_{0}\right)}} .
$$

Choosing $T_{0}=4.2 \mathrm{~K}, T=150 \mathrm{~K}$ and $\Theta_{\mathrm{D}}=248 \mathrm{~K}$, which are the temperatures at which our spectrum is a pure sextet, pure doublet so that $(n=2)$ and the Debye temperature of PIC, respectively, and using the Debye function [14] to calculate $f_{\mathrm{a}}(4.2 \mathrm{~K})$ and $\left.f_{\mathrm{a}}(150 \mathrm{~K})\right)$, the first factor on the RHS of eq. $(9 \mathrm{e})$ is 1.12 and all that would be present in the case of a single line; the second factor is 0.58 and reflects the collapse from the six-line to the two-line spectrum. The product is 0.65 . This implies that in the case of splitting with the above conditions, the apparent $f$-factor $f(T)$ decreases with increasing absorber thickness, which validates our original conclusion that at constant temperature, for large absorber thickness the apparent nor- 
malized $f$-factor is reduced from that for thin absorbers. Hence, one can conclude that there are $t w o$ competing effects that result from the thickness effect: the drop in the $f$-factor with increasing temperature weakens the anomaly, while the collapse of the spectrum with increasing temperature, due to superparamagnetism, enhances it. We cannot tell in advance which of these two effects will dominate. Therefore, it is evident that the absorber thickness effect must be taken into consideration.

The first experimental step in dealing with absorber thickness was to measure Mössbauer spectra for absorbers with varying thicknesses over the 4.2-300 K temperature range. This was done for PIC absorbers containing $10 \mathrm{mg} P I C / \mathrm{cm}^{2}$, $20 \mathrm{mg} \mathrm{PIC} / \mathrm{cm}^{2}, 50 \mathrm{mg} \mathrm{PIC} / \mathrm{cm}^{2}, 75 \mathrm{mg} \mathrm{PIC} / \mathrm{cm}^{2}$, and $100 \mathrm{mg} \mathrm{PIC} / \mathrm{cm}^{2}$, in addition to the initial $35 \mathrm{mg} \mathrm{PIC} / \mathrm{cm}^{2}$ absorber. When the absorption area for all absorbers and temperature was calculated and plotted (see fig. 2), we found, on the one hand, that there is an absorber thickness effect which seems to approach saturation for the thickest ( 75 and $100 \mathrm{mg}$ ) samples: the anomalous drop of the normalized area increases with thickness. On the other hand, the anomalous drop in the spectral area persists for the thinnest absorber. This is shown in fig. 2, where the normalized spectral area for the $10,35,75$, and $100 \mathrm{mg}$ absorbers are plotted versus temperature.

\subsubsection{Deconvolution of the effects of the Mössbauer absorber's thickness}

Various procedures [24-26] to correct for this effect have been given. However, they require assumptions about the source and absorber characteristics. The technique of Ure and Flinn [27] requires no assumptions about either source or absorber characteristics. The final result of this method is a spectrum which would be obtained from an ideally thin sample (absorber) and an ideally monochromatic source. Hence, it is the method of our choice.

We must note here, that in Mössbauer experiments with normalized spectra one does not determine directly the quantity $[N(\infty)-N(V) / N(\infty)]$, which we have used to determine the spectral area, but rather the quantity $\left[\epsilon(V)=(N(\infty)-N(V)) /\left(N(\infty)-N_{\mathrm{B}}\right)\right]$. Therefore, an accurate determination of the spectral area will involve a correction for the background radiation counting rate to the Mössbauer spectra. Many Mössbauer spectroscopists use the former quantity when only the relative variation of the $f$-factor or spectral area with temperature is desired. Thus, this background corrected Mössbauer spectrum is the one implied but not actually represented by most published spectra. However, the information desired from such a Mössbauer spectrum is $\sigma_{\mathrm{r}}(E)$, the resonant absorption cross-section from which one can determine the most accurate spectral area.

Figs. 4 and 5 show the original normalized Mössbauer transmission spectra and the deconvoluted spectra for the PIC $75 \mathrm{mg} / \mathrm{cm}^{2}$ and $10 \mathrm{mg} / \mathrm{cm}^{2}$ absorbers at temperatures $T=60$ and $85 \mathrm{~K}$ as a sample of all the absorber and various temperature spectra. The overextension of the right doublet line in fig. $5 \mathrm{~b}$ is due to effects 

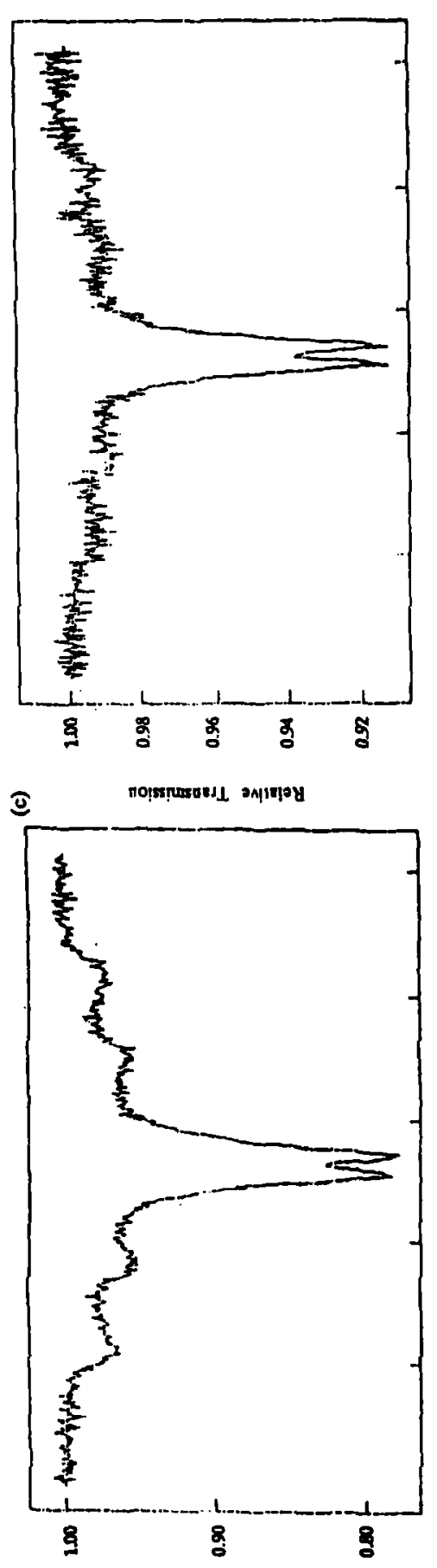

3

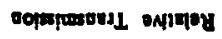
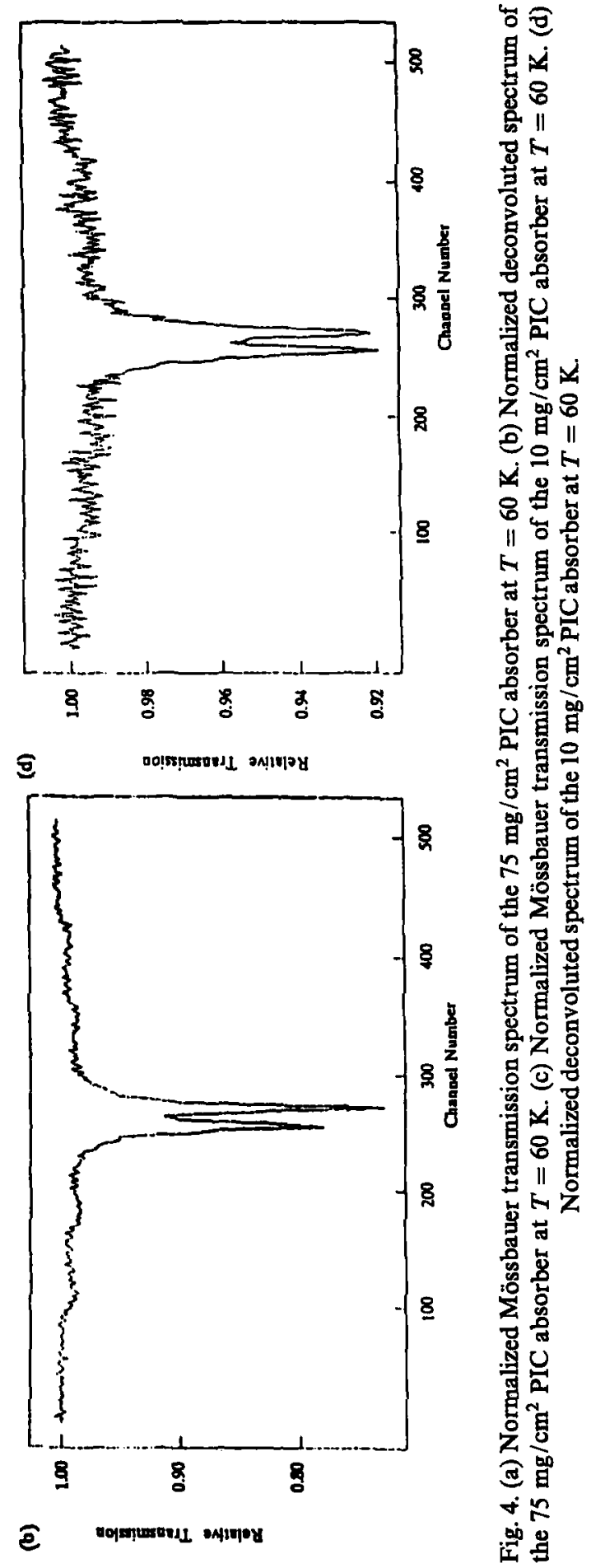

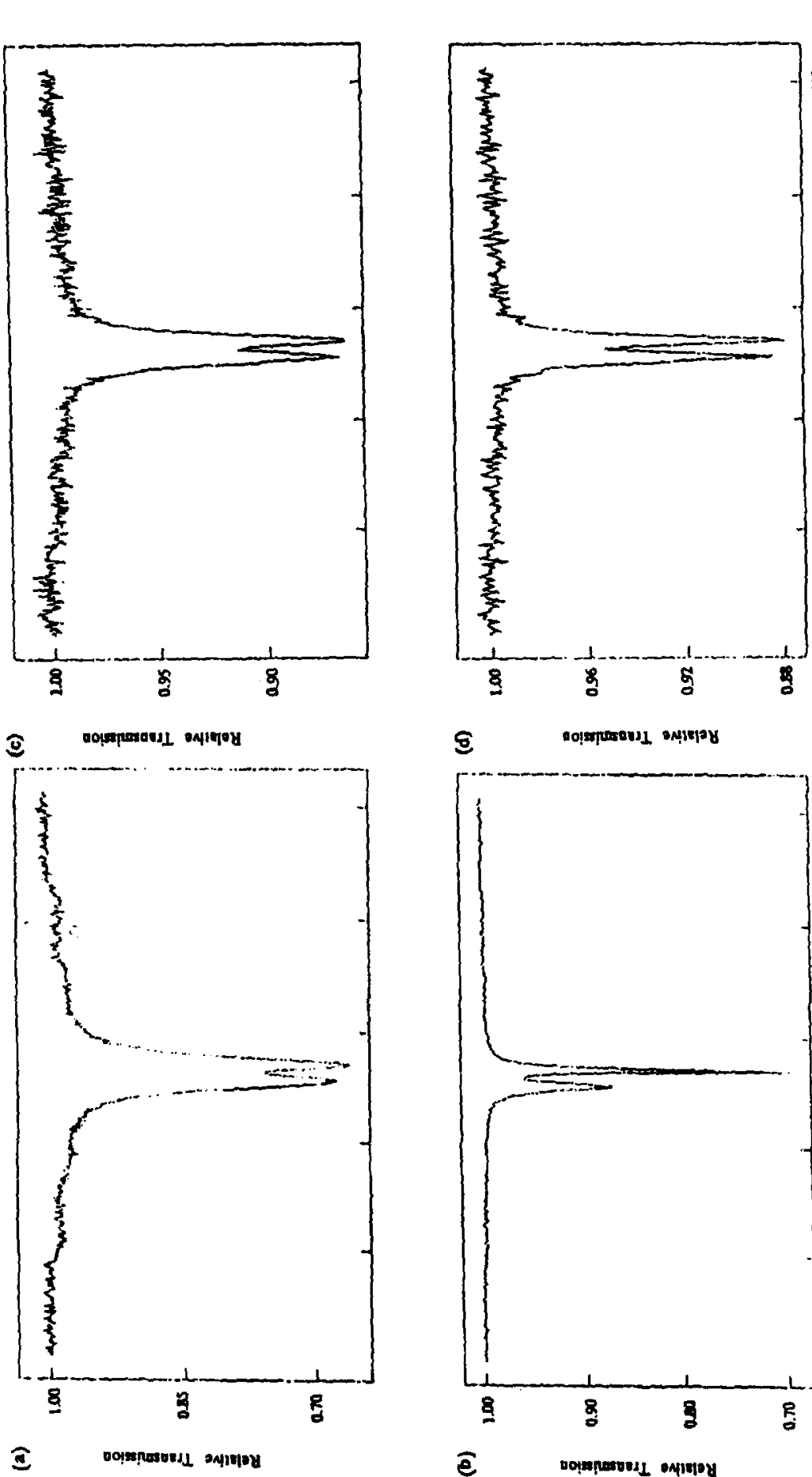

옹

慁

荧少

: II

75

当

递

$\frac{5}{2}$

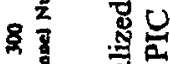

政

界的

$2 \div$

2!

$\therefore$

品

$\infty=$

811 岁要

$\rightarrow$ 目

ज्ञ

造

总总

突

兑首

․ㅡㅁ응

층

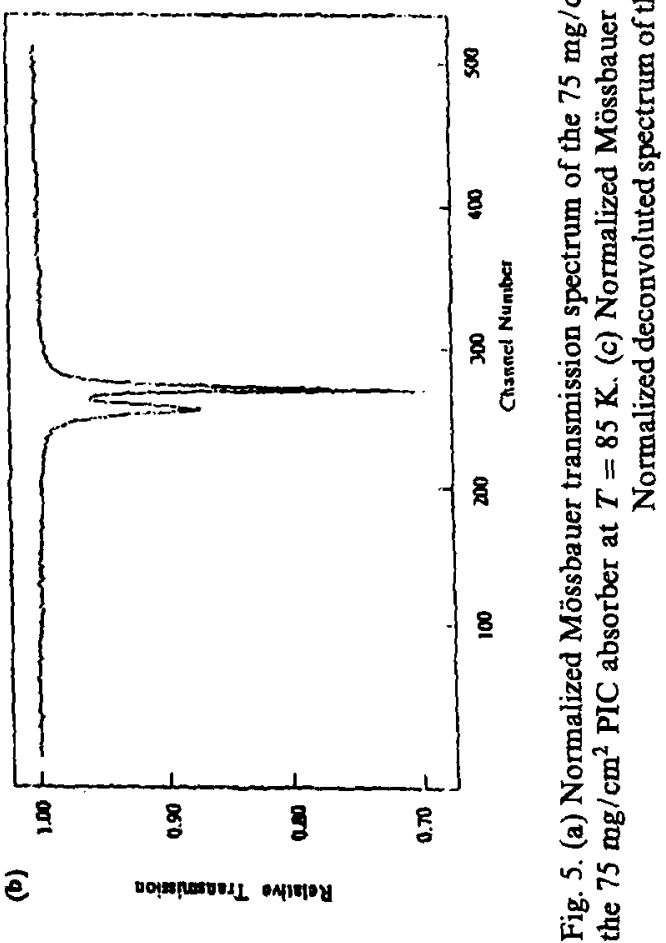


of the deviation (extreme scatter) of a couple of experimental spectral points on the Fourier transform, since in that region (the minima) the density of experimental points is not as high as in other regions of the spectrum. However, the deviant extension the effect of the doublet line on the spectral area is negligible.

The area of the absorption spectra for all PIC absorbers and all temperatures was evaluated from their deconvoluted spectra. All the corrected areas of all the absorbers fall within the range of experimental error of the $35 \mathrm{mg} / \mathrm{cm}^{2}$ absorber data. Hence, the "corrected" area, normalized to the $T=4.2 \mathrm{~K}$ value of the deconvoluted absorption spectrum of the $35 \mathrm{mg} / \mathrm{cm}^{2}$ PIC absorber, is plotted and shown in fig. 6 as a function of temperature. The solid curve in the figure represents a fit of the data of the Debye function (see section 6). It is clear that portions of the curve lie outside the error bars in the temperature range, $60-150 \mathrm{~K}$. As is evident from fig. 6 , the anomaly still persists after treatment and removal of the absorber thickness effects, which indicates that the source of the anomaly is not the absorber thickness.

\subsection{THEORETICAL FIT OF THE MÖSSBAUER SPECTRA OF PIC}

As indicated above, Mössbauer spectra were measured at eleven temperatures between 4.2 and $295 \mathrm{~K}$. The results of the absorber thickness effect analysis did not produce any appreciable change in going to the "thin" samples: $t_{\mathrm{a}}<3$ which is equivalent to the Mössbauer spectra of the $10 \mathrm{mg} / \mathrm{cm}^{2}$ absorber. The parameters from the fit of the Mössbauer spectra of the $10 \mathrm{mg} / \mathrm{cm}^{2}$ absorber are tabulated in table 1. At low temperatures, theoretical spectra were least-squares fitted to the experimental Mössbauer spectra (see fig. 6) with a combination of a distribution of

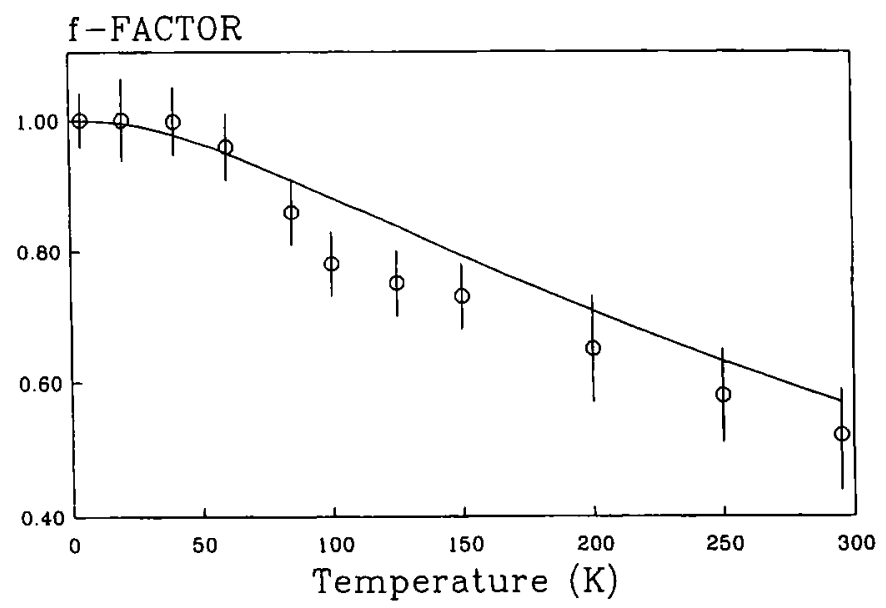

Fig. 6. The $f$-factor as a function of temperature for PIC. (O) represent the $f$-factor of the $35 \mathrm{mg} /$ $\mathrm{cm}^{2}$ PIC absorber, corrected for thickness effects versus temperature. The solid curve is a fit to experiment of the $f$-factor that is obtained from the Debye theory (eq. (22)). 
Table 1

Parameters of the theoretical spectra required to fit the $10 \mathrm{mg} / \mathrm{cm}^{2}$ PIC Mössbauer spectra at different temperatures.

\begin{tabular}{|c|c|c|c|c|c|c|}
\hline \multirow[t]{2}{*}{$T(\mathrm{~K})$} & \multirow{2}{*}{$\begin{array}{l}\text { Isomer } \\
\text { shift } \\
(\mathrm{mm} / \mathrm{s})\end{array}$} & \multicolumn{3}{|l|}{ SEXTET } & \multicolumn{2}{|l|}{ DOUBLET } \\
\hline & & $\begin{array}{l}\text { hyperfine } \\
\text { field (kOe) }\end{array}$ & $\begin{array}{l}\text { relative } \\
\text { area (\%) }\end{array}$ & $\begin{array}{l}\text { quadrupole } \\
\text { interaction } \\
2 E(\mathrm{~mm} / \mathrm{s})\end{array}$ & $\begin{array}{l}\text { quadrupole } \\
\text { splitting } \\
(\mathrm{mm} / \mathrm{s})\end{array}$ & $\begin{array}{l}\text { relative } \\
\text { area (\%) }\end{array}$ \\
\hline 4.2 & $0.45 \pm .008$ & $490^{\mathrm{a}}$ & 100 & & & \\
\hline 20 & $0.45 \pm .007$ & $451^{\mathrm{a}}$ & 89 & -0.09 & 0.90 & 11 \\
\hline 40 & $0.443 \pm .007$ & $400^{\mathrm{a}}$ & 76 & -0.09 & 0.89 & 24 \\
\hline 60 & $0.435 \pm .008$ & $350^{\mathrm{a}}$ & 55 & -0.07 & 0.87 & 45 \\
\hline \multirow[t]{3}{*}{85} & $0.43 \pm .008$ & 320 & 12 & -0.02 & 0.84 & 72 \\
\hline & & 260 & 8 & & & \\
\hline & & 160 & 8 & & & \\
\hline \multirow[t]{3}{*}{100} & $0.42 \pm .008$ & & & & 0.90 & 28 \\
\hline & & & & & 0.74 & 51 \\
\hline & & & & & 0.65 & 21 \\
\hline \multirow[t]{3}{*}{125} & $0.41 \pm .01$ & & & & 0.89 & 28 \\
\hline & & & & & 0.73 & 52 \\
\hline & & & & & 0.62 & 20 \\
\hline \multirow[t]{3}{*}{150} & $0.392 \pm .009$ & & & & 0.90 & 21 \\
\hline & & & & & 0.72 & 54 \\
\hline & & & & & 0.60 & 25 \\
\hline \multirow[t]{3}{*}{200} & $0.36 \pm .008$ & & & & 0.91 & 16 \\
\hline & & & & & 0.73 & 62 \\
\hline & & & & & 0.59 & 22 \\
\hline \multirow[t]{3}{*}{250} & $0.33 \pm .009$ & & & & 0.92 & 18 \\
\hline & & & & & 0.72 & 59 \\
\hline & & & & & 0.57 & 23 \\
\hline 295 & $0.301 \pm .008$ & & & & $0.72^{b}$ & \\
\hline
\end{tabular}

Maximum of a distribution of hyperfine fields, see figs. 9a, $9 \mathrm{~b}$, and $9 c$.

b Maximum of a distribution of QS, see fig. 9d.

sextets and a doublet. The percent of each contributed spectrum, i.e. the sextet distribution and the doublet, is given in table 1. Our fitting programs were of two types: Type (A) fits the spectra with a finite number of magnetic hyperfine fields, quadrupole splitting electric field gradients, or both. The other type (B) fits the spectra with a broad distribution of hyperfine fields (with or without a quadrupole), or a broad distribution of quadrupoles. The spectra at low temperatures are obviously (fig. 7) broadened sextuplets with asymmetric lines due to a distribution of hyperfine fields, which is validated by the good fits we achieved using a distribution of sextets. The spectra at intermediate temperatures can be fitted by a smaller number of sextets than the low-temperature spectra. At $T=85 \mathrm{~K}$, the fit was achieved with three sextets and one doublet, however, for higher temperatures the use of three doublets gave the best fit, see fig. 8 . The contribution from the doublet increases with temperature as expected from a distribution of small particle sizes. 
(a)

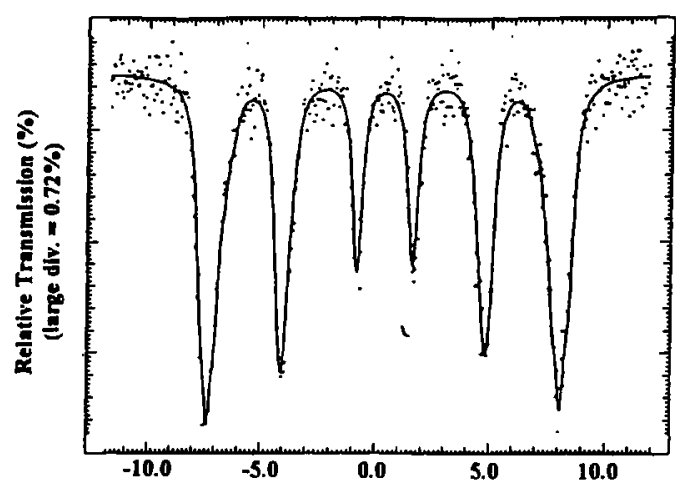

(b)

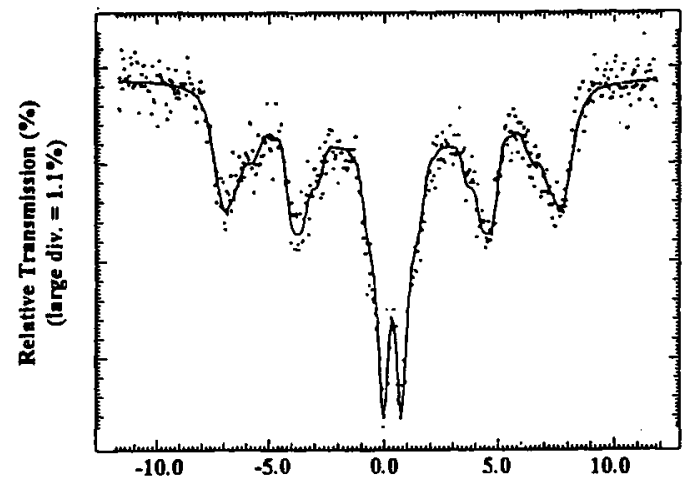

(c)

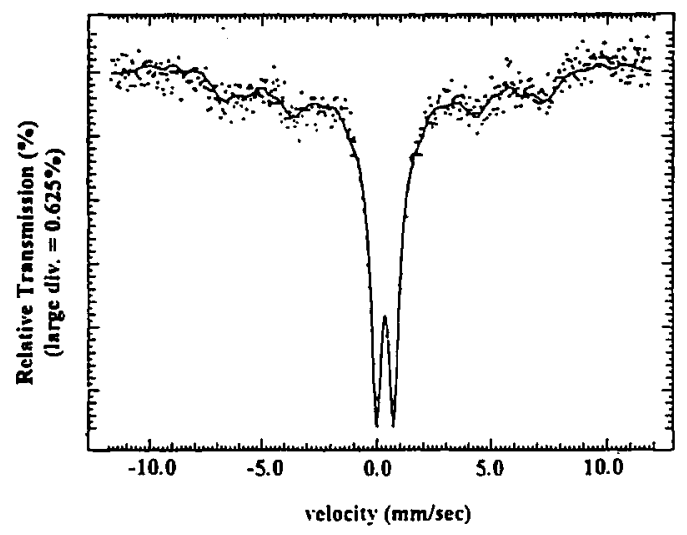

Fig. 7. Least-squares fit of the original normalized $10 \mathrm{mg} / \mathrm{cm}^{2}$ PIC Mössbauer spectra at (a) $T=4.2 \mathrm{~K}$; (b) $T=40 \mathrm{~K}$; (c) $T=60 \mathrm{~K}$. The deconvoluted and original Mössbauer spectra for the $10 \mathrm{mg} / \mathrm{cm}^{2}$ PIC absorber showed little appreciable difference.

The probability distribution, $P(H)$, of the hyperfine fields for the temperatures $T=4.2,20$ and $40 \mathrm{~K}$ is shown in figs. $9 \mathrm{a}, 9 \mathrm{~b}$ and $9 \mathrm{c}$. All exhibit sharp maxima. Some oscillations with occasional negative contributions to $P(H)$ appear in fig. 9. These are of no physical consequence and usually result from the limit imposed on 
(a)

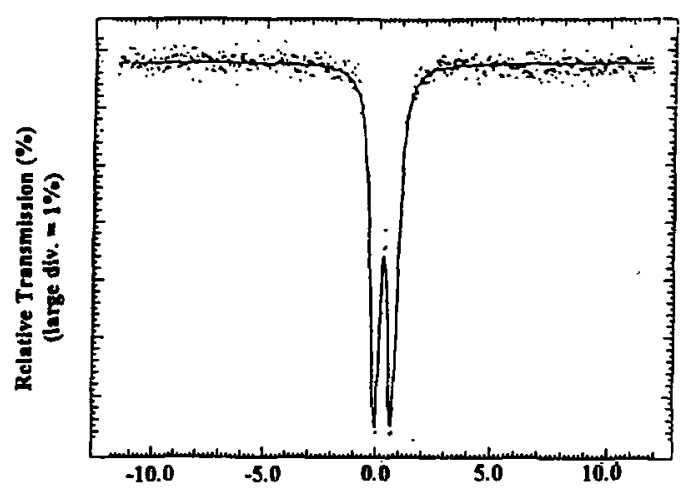

(b)

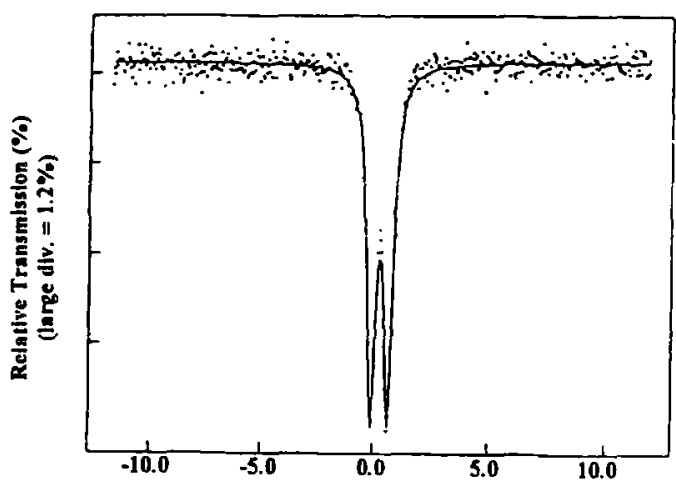

(c)

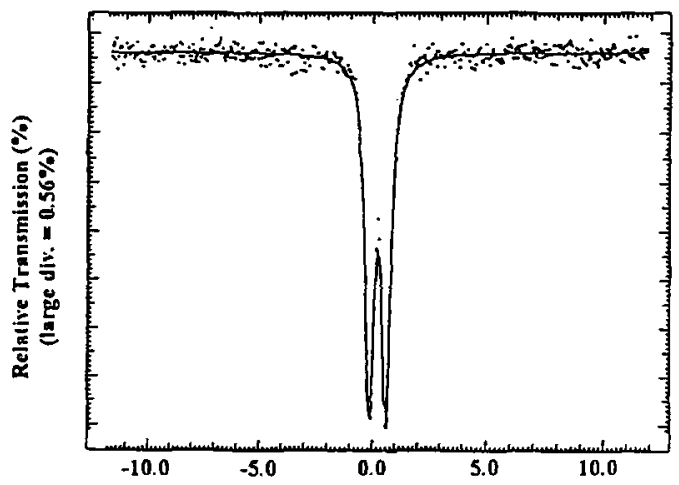

Fig. 8. Least-squares fit of the original normalized $10 \mathrm{mg} / \mathrm{cm}^{2}$ PIC Mössbauer spectra at (a) $T=200 \mathrm{~K}$; (b) $T=250 \mathrm{~K}$; (c) $T=295 \mathrm{~K}$. The deconvoluted and original Mössbauer spectra for the $10 \mathrm{mg} / \mathrm{cm}^{2}$ PIC absorber showed little appreciable difference.

the distribution increment and from statistical fluctuations in the experimental data. On the low side of the maxima in figs. $9 \mathrm{a}-9 \mathrm{c}$ the slopes are less steep than on the high side. For $T=295 \mathrm{~K}$, a distribution of $P(Q)$ of these doublets is shown in fig. $9 \mathrm{~d}$. The quadrupole splitting of the doublet appears to vary with temperature 
(a)

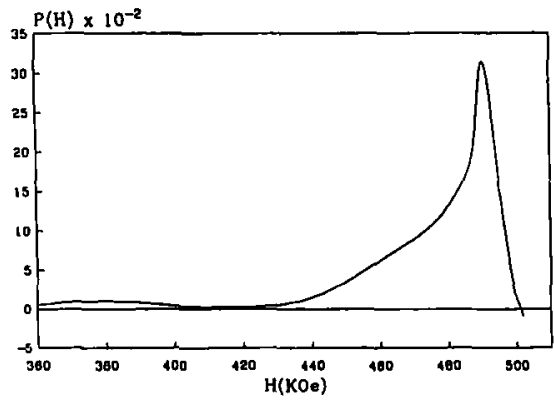

(c)

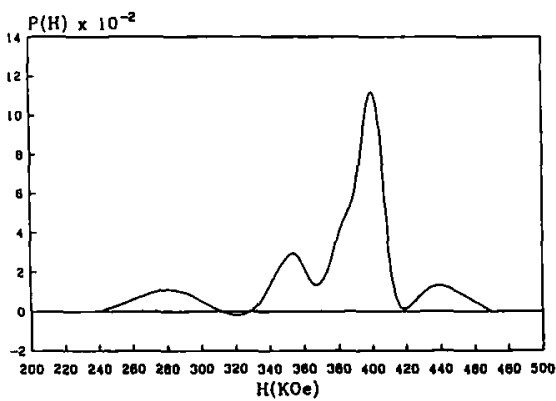

(b)

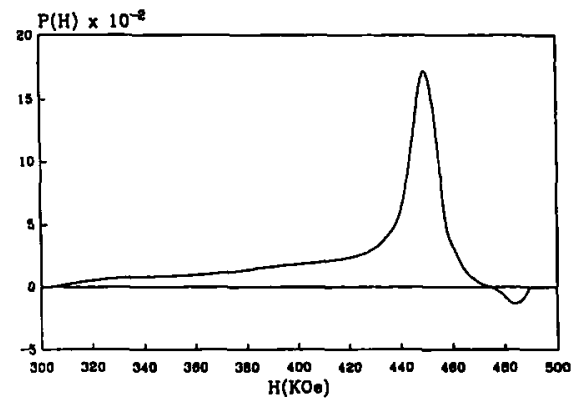

(d)

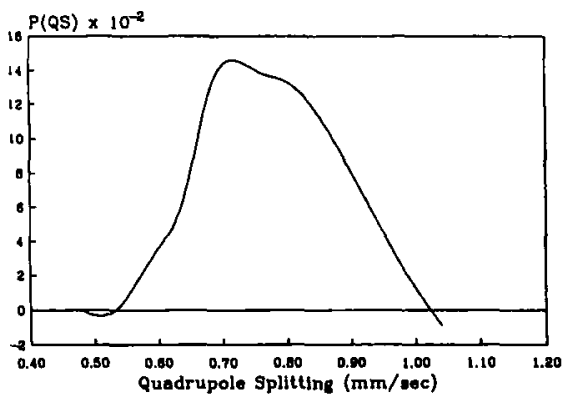

Fig. 9. The magnetic hyperfine field distribution $P(H)$ obtained from the theoretical fit of the Mössbauer spectrum of the $10 \mathrm{mg} / \mathrm{cm}^{2}$ PIC absorber at (a) $T=4.2 \mathrm{~K}$, (b) $20 \mathrm{~K}$, (c) $40 \mathrm{~K}$. (d) The electric quadrupole splitting distribution $P(Q)$ obtained from the theoretical fit of the Mössbauer spectrum of the $10 \mathrm{mg} / \mathrm{cm}^{2}$ PIC absorber at $T=295 \mathrm{~K}$.

over the lower temperature range, due to relaxation processes, as will be elucidated in the following section. However, over the remaining temperature range, the quadrupole splitting is relatively constant, as expected. In fact, we expect the $T=200$ and $250 \mathrm{~K}$ spectra (figs. $8 \mathrm{a}$ and $8 \mathrm{~b}$ ), which were fitted with a type (A) fitting program, to yield a similar distribution and peak value to that obtained for the $T=295 \mathrm{~K}$ spectra (fig. 8c), if fitted with a type (B) program, which the $T=295 \mathrm{~K}$ spectra was fitted with. In the case of a combined magnetic and quadrupole interaction where the quadrupole interaction is much weaker than the magnetic one (i.e. at low $T$ ), the quadrupole interaction can be treated as a small perturbation to the magnetic interaction. Hence, all the magnetic hyperfine field lines are shifted by a quantity [14]

$$
\frac{1}{8} e^{2} q Q\left(3 \cos ^{2} \psi-1+\eta^{2} \sin ^{2} \psi \cos 2 \phi\right),
$$

where $e$ is the charge of the proton, $e Q$ is the nuclear electric quadrupole moment, $e q$ is the maximum value of the electric field gradient (EFG) tensor along its principal axis at the Mössbauer nucleus and $\phi$ and $\psi$ are polar angles describing the orien- 
tation of the principal axis of the EFG with respect to the magnetic hyperfine field direction. If the asymmetry parameter $\eta(0 \leqslant \eta \leqslant 1)$ is equal to zero, which we expect it to be for the EFG at the nucleus of the high spin $(S=5 / 2) \mathrm{Fe}^{3+}$ ion in PIC and ferritin, then the above quantity is simplified. Then, the difference between the sixth and fifth lines and that of the second and first lines of the sextet yields the quadrupole interaction $2 \varepsilon=\left(e^{2} Q / 2\right)\left(3 \cos ^{2} \psi-1\right) / 2$ in this low temperature range, which is different from the observed quadrupole splitting at higher temperatures: $\mathrm{QS}=e^{2} Q / 2$ (i.e. $\left.\mathrm{QS}=e^{2} q Q / 2\left(c / E_{0}\right)(\mathrm{mm} / \mathrm{s}), E_{0}=14.4 \mathrm{keV}\right)$. Our results yield an average value of $58^{\circ}$ for $\psi$.

The isomer shift varies with temperature (see table 1) due to the second-order Doppler shift and corresponds to high-spin $\mathrm{Fe}^{3+}[14]$. Using the definition of the blocking temperature $T_{\mathrm{B}}$ as the temperature at which the sextet and doublet contributions to the Mössbauer spectra are equal, we find $T_{\mathrm{B}}=63 \mathrm{~K}$ for PIC. The blocking temperature for human ferritin derived similarly from Mössbauer spectra is $T_{\mathrm{B}}=40 \mathrm{~K}$ [5], while that for horse spleen ferritin has been reported as $T_{\mathrm{B}}=37 \mathrm{~K}$ [6], and has been shown, recently, to vary with the degree of iron loading [28].

The fits that we obtained with the Mössbauer spectra were good. A plot of the fractional residuals for all spectra and temperatures versus velocity $(\mathrm{mm} / \mathrm{s})$ produced featureless scatter plots, which is the best graphical way of showing goodness of fit. In figs. $10 \mathrm{a}$ and $10 \mathrm{~b}$ examples of such plots for the Mössbauer spectra of the $10 \mathrm{mg} / \mathrm{s}^{2}$ absorber at $T=4.2 \mathrm{~K}$ and $T=125 \mathrm{~K}$ are shown. It is clear from the figures that the residuals are not on one side of the zero-line in any resonant frequency or velocity vicinity, but rather are featurelessly scattered about the zero-line and throughout the range of velocities.

\subsubsection{Correlation between hyperfine interactions and particle sizes}

For particles with volumes $V$ and uniaxial symmetry, the magnetic crystalline energy is given by $E(\theta)=K V \sin ^{2} \theta$, where $\theta$ is the angle between the magnetiza-

(a)

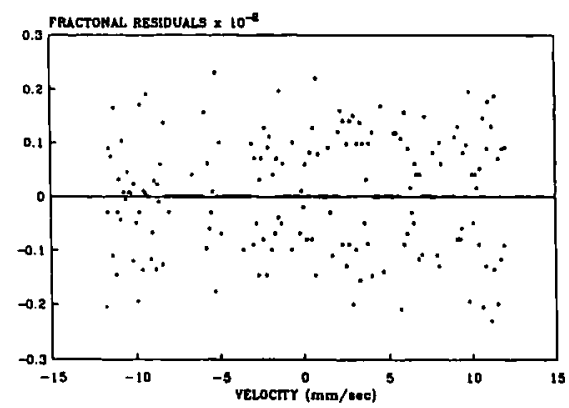

(b)

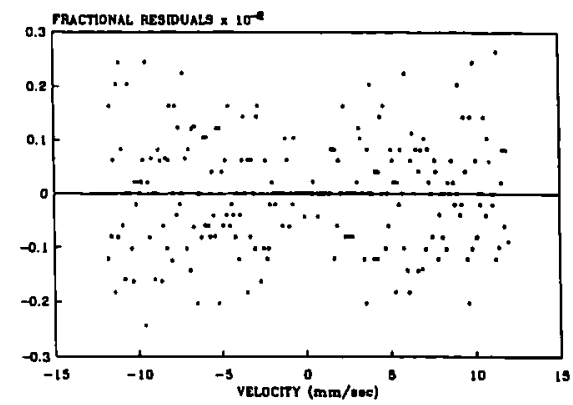

Fig. 10. Fractional residuals: ((theor. fit - experiment)/theor. fit) versus velocity (mm/s) for the Mössbauer spectrum of the $10 \mathrm{mg} / \mathrm{cm}^{2}$ PIC absorber at (a) $T=4.2 \mathrm{~K}$ and (b) $T=125 \mathrm{~K}$. 
tion vector $M$ and the easy direction $\Delta$ of magnetization and $K$ is the anisotropy constant. The usual model of superparamagnetism describes the relaxation process in terms of transitions between the two states; $\theta=0$ and $\theta=\pi[29,30]$. However, this model failed to explain the asymmetric line broadening occurring far below the blocking temperature. Mørup et al. [31,32] introduced collective magnetic excitations which result in fluctuations of the magnetization $M$, and hence the hyperfine field $H$, around the easy direction $\Delta$ so that for a particle size $V$ and at temperature $T$, a nucleus will experience a thermally averaged field given by

$$
H(V, T)=H(\infty, T)\langle\cos \theta\rangle_{T},
$$

where $\langle\cos \theta\rangle_{T}$ is expressed through

$$
\langle\cos \theta\rangle_{T}=\frac{\int_{0}^{\pi / 2} \exp -\left[E(\theta) / K_{\mathrm{B}} T\right] \cos \theta \sin \theta \mathrm{d} \theta}{\int_{0}^{\pi / 2} \exp -\left[E(\theta) / K_{\mathrm{B}} T\right] \sin \theta \mathrm{d} \theta} .
$$

Williams et al. [33] extended Mørup's model to cases including both hyperfine field and quadrupole interaction fluctuations, where he considered a frame of reference in which the easy direction of magnetization $\Delta$ is along the $z$-axis, the principal axis of the electric field gradient EFG is in the $y z$-plane, the projection of $H$ on to the $x y$-plane is at an angle $\phi$ with respect to the $x$-axis and the angle between $H$ and EFG is $\psi$, while $\theta$ is the angle between $H$ and $\Delta$.

At very low temperatures, if the quadrupole interaction may be considered as a weak perturbation in the Hamiltonian, the nuclear level splitting will be given by the well known approximation $[14,33]$

$$
E_{m}=-g \mu_{\mathrm{N}} H m+A\left[3 m^{2}-j(j+1)\right] \frac{1}{2}\left(3 \cos ^{2} \psi-1\right),
$$

where $g$ is the nuclear gyromagnetic ratio, $\mu_{\mathrm{N}}$ is the nuclear magneton, $j$ is the nuclear spin, $m(=j, j-1, \ldots,-j)$ is the spin projection along the direction of the magnetic hyperfine field $H, A$ is the quadrupole coupling constant and $\psi$ is the angle between $H$ and EFG as defined above. If $H$ is fluctuating around $\Delta$, this will result in an apparent hyperfine field:

$$
\langle H\rangle=H_{0}\langle\cos \theta\rangle_{T} .
$$

A straightforward calculation [30] using eq. (12) gives an expression for $\langle\cos \theta\rangle_{T}$ :

$$
\langle\cos \theta\rangle_{T}=\frac{\int_{0}^{\pi / 2} \exp \left(a \cos ^{2} \theta\right) \cos \theta \sin \theta \mathrm{d} \theta}{\int_{0}^{\pi / 2} \exp \left(a \cos ^{2} \theta\right) \sin \theta \mathrm{d} \theta}=\frac{1}{2 a^{1 / 2}} \frac{1-\exp (-a)}{F\left(a^{1 / 2}\right)},
$$

where

$$
a=K V / K_{\mathrm{B}} T, F\left(a^{1 / 2}\right)=\exp (-a) \int_{0}^{a^{1 / 2}} \exp \left(x^{2}\right) \mathrm{d} x .
$$

$F\left(a^{1 / 2}\right)$ is the so-called Dawson's integral, numerical values of which are available 
[34] or can be easily calculated. However, $\langle\cos \theta\rangle_{T}$ can be quite well expressed by two asymptotic relations with an error always smaller than a few per cent that is

$$
\langle\cos \theta\rangle_{T}= \begin{cases}1-1 / 2 a & a>5, \\ \frac{1}{2}+\frac{1}{12} a & 1<a<5 .\end{cases}
$$

Using these asymptotic expressions, the apparent $H(V, T)$ is given by Williams et al. [33] as

$$
H(V, T)= \begin{cases}H(\infty, T)\left(1-K_{\mathrm{B}} T / 2 K V\right) & a=K V / K_{\mathrm{B}} T>5, \\ H(\infty, T)\left(\frac{1}{2}+K V / 12 K_{\mathrm{B}} T\right) & 1<a=K V / K_{\mathrm{B}} T<5 .\end{cases}
$$

At very high temperatures, the remaining hyperfine field, if it still exists, will be a weak perturbation in the Hamiltonian compared to the quadrupole interaction, and the nuclear splitting will be

$$
E_{m}=A\left[3 m^{2}-j(j+1)\right]-g \mu_{\mathrm{N}} H M \cos \psi .
$$

Taking into account possible fluctuations, the apparent hyperfine field is given by [33]

$$
\langle H\rangle=H_{0}\langle\cos \psi\rangle_{T} .
$$

However, in most cases, in this temperature range (above the blocking temperature) according to conventional superparamagnetic relaxation, the magnetization vector of the particle will jump between the energy minima at $\theta=0$, and $\pi$, at such a rate, $\lambda\left(1 / \lambda \leqslant 2.5 \times 10^{-9} \mathrm{~s}\right)$, that the average magnetization $\langle M\rangle$ and hence the observed hyperfine field will vanish. On the other hand, below $T_{B}$ the relaxation of the magnetization vector among the easy directions can be considered negligible. The fluctuations of the magnetization vector $M$ in directions close to an easy direction are fast compared to the time scale of Mössbauer spectroscopy $\left(\approx 10^{-9} \mathrm{~s}\right)$. Hence, the observed magnetic hyperfine field at the nucleus is considered proportional to the average magnetization $\langle M\rangle$, which leads to the results at the beginning of this section.

If generally, the magnetic energy of the particles is more complicated than that of uniaxial magnetic symmetry, it is then convenient to express [32] the magnetic energy of the particle as a function of the direction cosines $u_{x} u_{y} u_{z}$ of the magnetization vector: $E=E\left(u_{x} u_{y} u_{z}\right)$. Below $T_{\mathrm{B}}$, the magnetization vector $M$ remains near one of the easy directions (defining the $z$-direction) with a finite probability that it forms a small angle with the easy direction. As shown by Mørup [32] an asymptotic relation for the average hyperfine field at low temperatures is

$$
\langle H\rangle=H_{0}\left\{1-\frac{1}{2} K_{\mathrm{B}} T\left[\left(\frac{\partial^{2} E}{\partial u_{x}^{2}}\right)^{-1}+\left(\frac{\partial^{2} E}{\partial u_{x}^{2}}\right)^{-1}\right]\right\} .
$$

This implies, as Mørup has pointed out, that this collective magnetic excitation the- 
ory, as manifested by the changes of the hyperfine field with temperatures, can yield a value of $K V$ (more precisely a value of $K$ ) based on the assumption of a particular form of magnetic energy, e.g. uniaxial. However, if $K$ obtained from this theory differs from the values of $K$ derived independently from (superparamagnetic) relaxation spectra or magnetic data by an amount greater than $20 \%$, then one must assume that the magnetic energy of the particle is more complicated than the assumed magnetic energy with uniaxial symmetry. For example, the particle may have two or more easy axes due possibly to the contribution of shape and/or exchange anisotropy.

From the values of the magnetic hyperfine field at different temperatures (fig. 9), we see that the cut-off value $H_{\max }=H_{0}$ for the $T=4.2 \mathrm{~K}$ hyperfine field distribution is $500 \mathrm{kOe}$. Hence $H_{0}=500 \mathrm{kOe}$ will be identified as $H(\infty, T)$ in eqs. (14) and (16). Using eqs. (14), (15), and (16) to fit our normalized hyperfine field values $\left(H / H_{0}\right)$ obtained from the theoretical fits of the spectra, we obtain a value of $1.9 \times 10^{-14} \mathrm{erg}$ for $K V$. Fig. 11 shows the result of this fit, where the curve in the figure represents eqs. (14) and (15) while the $\times$ points and the straight lines represent the asymptotic relations given in eq. (16).

For a distribution of superparamagnetic particle volumes, there will exist a distribution of magnetic hyperfine field values at the Mössbauer nuclei, mainly due to the existence of different environments for these nuclei, i.e. in bulk or surface atoms in the particle and also due to collective magnetic excitations. Consequently, it is necessary to operate at sufficiently low temperatures to make sure of having a pure hyperfine field distribution $P(H)$ to enable us to determine the particle size distribution. At intermediate temperatures, the spectra are fitted with a single doublet and a distribution of hyperfine fields $P(H)$. These $P(H)$ curves are more

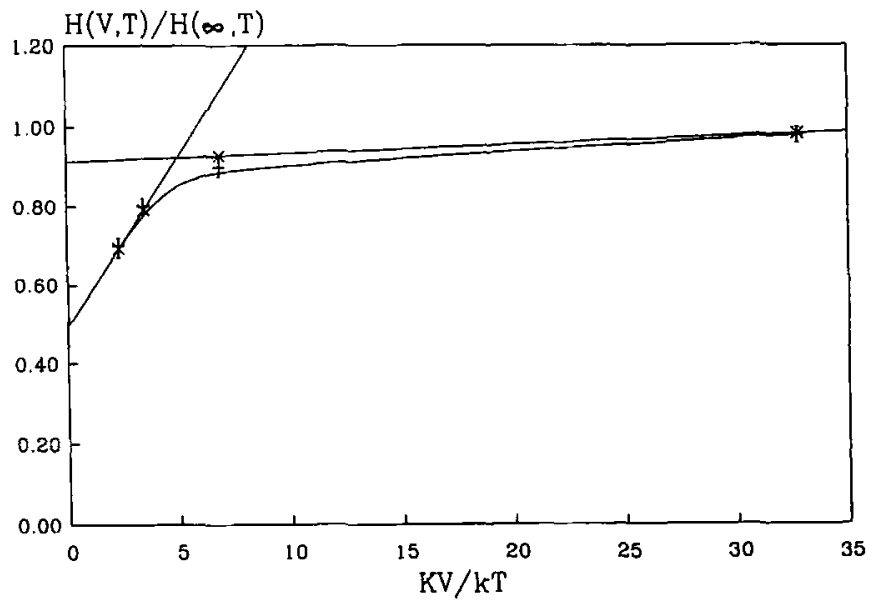

Fig. 11. The normalized magnetic hyperfine field versus $a=K V / K_{\mathrm{B}} T$. The curve represents eqs. (11) and (15). The $(x)$ and the straight lines representing the asymptotic expressions in eq. (16) fit the experimental points with a value of $K V=1.9 \times 10^{-14} \mathrm{erg}$. 
poorly defined than those at the lowest temperatures. This arises from the fact that the doublet and $P(H)$ overlap and a fraction of the spectral central component can be analyzed in terms of a hyperfine field, so, giving an artificial contribution to $P(H)$. Therefore, at $T=4.2 \mathrm{~K}$, we are sure of obtaining such a pure $P(H)$ which should correspond to particle size spread with a maximum diameter not greater than $120 \AA$ and a minimum no less than $30 \AA$ based on the comparison of PIC's and ferritin's $[33,16]$ sextet/doublet areas and the distribution of particle volumes for ferritin [33].

Before we are able to determine the particle size distribution we must estimate the value of the anisotropy constant $K$ for our sample of PIC. Using the asymptotic relations eq. (16) with $a=K V / K_{\mathrm{B}} T=K \pi d^{3} / 6 K_{\mathrm{B}} T, K V=1.9 \times 10^{-14} \mathrm{erg}, T=$ $4.2 \mathrm{~K}$, to fit the values of the maximum and minimum normalized hyperfine field values $\left(H / H_{0}\right)$ of the $T=4.2 \mathrm{~K}$ hyperfine field distribution yields $K=1.06$ $\times 10^{5} \mathrm{erg} / \mathrm{cm}^{3}$. We note that the value of $K$ obtained from magnetic data on PIC is $K \approx 3 \times 10^{5} \mathrm{erg} / \mathrm{cm}^{3}$ [20]. The difference $(\sim 60 \%)$ between these values of PIC's anisotropy constant indicates that its magnetic anisotropy energy is not simply uniaxial. One should also note that Williams et al. [33], using the collective magnetic excitations theory in conjunction with the assumption of uniaxial symmetry, has found $K=0.7 \times 10^{5} \mathrm{erg} / \mathrm{cm}^{3}$ for ferritin, while St. Pierre [35] has argued convincingly using ferritin Mössbauer spectra in applied magnetic fields, that $K \geqslant 2 \times 10^{5} \mathrm{erg} / \mathrm{cm}^{3}$ for ferritin. We have found $K=2.7 \times 10^{5} \mathrm{erg} / \mathrm{cm}^{3}$ for naturally occurring (mostly $\mathrm{Fe}^{3+}$ ) ferritin [20] from magnetization measurements.

\subsubsection{Particle size distribution}

To calculate the probability $P(d)$ of having a particle of diameter $d$, using the equivalent $P(H)$ hyperfine distribution curve, a renormalization process must be undertaken to allow for the non-linear transformation of the coordinate axis from $H(\mathrm{kOe})$ to $d(\AA)$. We accomplished this process by utilizing the following procedure used by Williams et al. [33]:

- The range of diameters expected is divided into equal steps of length $s$, a typical step centering on a diameter $d_{\text {cent }}$ and with external values $d_{\text {ex } 1}=d_{\text {cent }}-(s / 2)$, $d_{\mathrm{ex} 2}=d_{\text {cent }}+(s / 2)$.

- For each $d_{\mathrm{ex}}$, the value of $a$ is calculated from $a=K \pi d^{3} / 6 K_{\mathrm{B}} T$ using the value $K=1.06 \times 10^{5} \mathrm{erg} / \mathrm{cm}^{3}$ estimated in the previous section.

- Using the value of $a$ and eqs. (14) and (15) in the form

$$
H_{\mathrm{ex}}=H_{0}\left(\frac{1}{2 a^{1 / 2}} \frac{1-\exp (-a)}{F\left(a^{1 / 2}\right)}\right)
$$

and calculating the Dawson integral $F\left(a^{1 / 2}\right)$ numerically, using Simpson's rule, the field $H_{\text {ex }}(\mathrm{kOe})$ corresponding to each $d_{\mathrm{ex}}$ is determined, taking $H_{0}=500 \mathrm{kOe}$ (see previous section).

- Next, the area under the $P(H)$ against $H$ curve (i.e. the $P(H, T=4.2 \mathrm{~K}$ ) 
curve) is calculated for each range $H_{\mathrm{ex} 1}$ to $H_{\mathrm{ex} 2}$ (i.e. equivalent to the step in diameters of length $s$, centering on $\left.d_{\text {cent }}\right)$. This area gives the probability $P\left(d_{\text {cent }}\right)$ of finding a diameter $d_{\text {cent }}$.

- Finally, $P(d)$ is normalized in a way such that $\sum P(d)=100$.

This process leads to numerical values of $d, P(d)$, and $\sum P(d)$, as well as to the particle size distribution curve shown in fig. 12. $\sum P(d)$ gives the proportion of particles smaller than a given $d$ value. The distribution curve yields an average particle diameter, $d=70 \AA$, for our PIC absorber which is slightly larger than the value for ferritin $d \approx 62 \AA$ or that given for ferritin by Williams et al. [33], $d \approx 65 \AA$. This is expected since the sextet components for our PIC spectra, as well as that of Berg et al. [16], are larger than that of ferritin [33] at higher temperatures, which indicates a larger average particle size for PIC than for the ferritin used by Williams et al. [33].

\section{Influence of core (particle) motion on the Mössbauer effect}

Two arguments have been proposed for the observed drop in the $f$-factor. We have shown that the thickness effect is not the cause of the anomalous $f$-factor, though it can accentuate it. Another source that we considered is the vibrations of the core as a whole. It has been proposed $[14,36]$ that the $f$-factor is given by the product

$$
f(T)=f_{0}(T) f_{1}(T),
$$

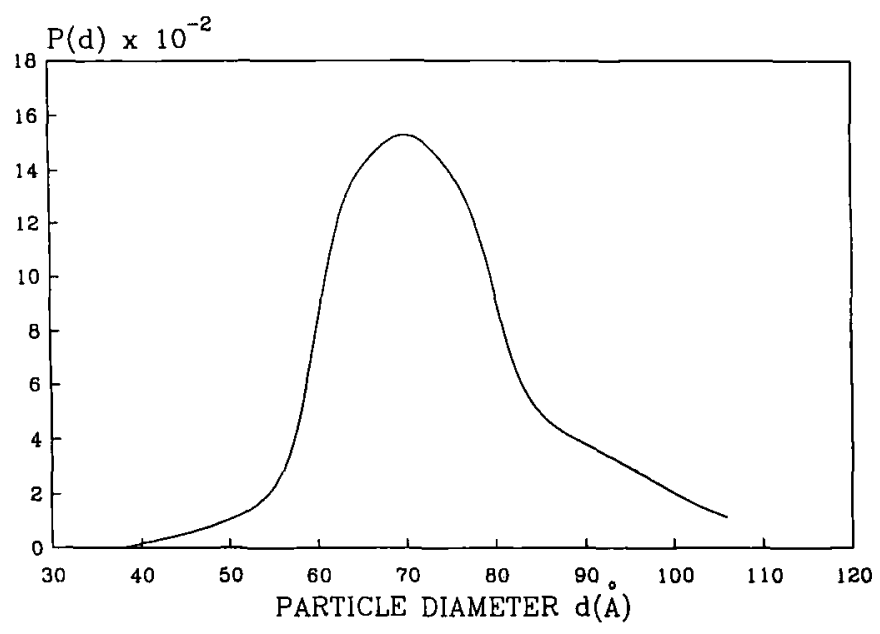

Fig. 12. The distribution of particle diameters $(\AA): P(d)$ obtained from the hyperfine field distribution of the Mössbauer spectrum of the $10 \mathrm{mg} / \mathrm{cm}^{2}$ PIC absorber at $T=4.2 \mathrm{~K}$. See ref. [33] for details. 
where $f_{0}=\exp \left[-k^{2}\left\langle x_{0}^{2}\right\rangle_{T}\right]$ reflects the displacement $x_{0}$ of the Mössbauer nucleus with respect to the lattice and $f_{1}=\exp \left[-k^{2}\left\langle x_{1}^{2}\right\rangle_{T}\right]$ reflects the displacement of the core with respect to the protein shell. Hence, the msd of the Mössbauer effect atom is expressed as

$$
\left\langle x^{2}\right\rangle_{T}=\left\langle x_{0}^{2}\right\rangle_{T}+\left\langle x_{1}^{2}\right\rangle_{T}
$$

where $f(T)=\exp \left[-k^{2}\left\langle x^{2}\right\rangle_{T}\right]$.

In general, superparamagnetic particles as a whole may vibrate. In Mössbauer spectroscopy of small particles, the $f$-factor, as measured by the resonant absorption area, is often smaller than in the corresponding bulk materials. This effect has been observed for microcrystals of gold [36], tin [37], iron [38], and iron oxide [39]. Viegers and Trooster [36] suggested the above relation eq. (20), where $f_{0}(T)$ contains information about the particle phonon spectrum, while $f_{1}(T)$ gives information about the binding of the particle to the medium. The validity of this approach was demonstrated by Hayashi et al. [38], who measured the resonant absorption areas of $100 \AA$ iron particles in various matrices. Hayashi's method, in which $f(T)$ of free particles is compared with that of embedded particles, yields indirect, although convincing, evidence of particle motion only. Niemantsverdriet [40] has demonstrated that the presence of particle motion can be recognized from the Mössbauer spectra of small particles, by determining the temperature dependence of both the recoilless fraction and the second order Doppler shift, the latter indicated by the isomer shift IS $(T)$. Both $f(T)$ and IS $(T)$ can be expressed in the Debye model as a function of $\theta_{\mathrm{D}}$, the Debye temperature and $T$. The expressions are $[41,42]$

$$
f(T)=\exp \left\{-\frac{6 E_{\mathrm{R}}}{K_{\mathrm{B}} \Theta_{\mathrm{D}}}\left[\frac{1}{4}+\left(\frac{T}{\theta_{\mathrm{D}}}\right)^{2} \int_{0}^{\theta_{\mathrm{D}} / T} \frac{x}{e^{x}-1} \mathrm{~d} x\right]\right\} .
$$

and

$$
\operatorname{IS}(T)=\operatorname{IS}(0)-\frac{9}{2}\left(\frac{K_{\mathrm{B}} T}{M c}\right)\left(\frac{T}{\theta_{\mathrm{D}}}\right)^{3} \int_{0}^{\theta_{\mathrm{D}} / T} \frac{x^{3}}{e^{x}-1} \mathrm{~d} x,
$$

in which $E_{\mathrm{R}}=1.9 \times 10^{-3} \mathrm{eV}=E_{\gamma}^{2} / 2 M c^{2}$ is the recoil energy of the ( $E_{\gamma}=$ $14.4 \mathrm{keV}) \gamma$-ray, $M$ is the mass of the ${ }^{57} \mathrm{Fe}$ nucleus, $K_{\mathrm{B}}$ is Boltzmann's constant, and $c$ is the velocity of light. For thin absorbers (or absorber whose thickness effect has been corrected), the resonant absorption area, $A(T)$, of the Mössbauer spectrum is proportional to $f$ and hence $A_{\mathrm{N}}(T)=A(T) / A\left(T_{0}\right)=f(T) / f\left(T_{0}\right)=f_{\mathrm{N}}(T)$, where $\mathrm{N}$ indicates normalization to the $T_{0}$ data. For our PIC data $T_{0}=4.2 \mathrm{~K}$. Therefore in the high temperature range $\left(T>\theta_{\mathrm{D}} / 2\right)$, this leads to

$$
\frac{\mathrm{d}\left(\ln A_{\mathrm{N}}\right)}{\mathrm{d} T}=\frac{\mathrm{d}\left(\ln f_{\mathrm{N}}\right)}{\mathrm{d} T} \rightarrow-\frac{6 E_{\mathrm{R}}}{K_{\mathrm{B}} \theta_{\mathrm{D}}^{2}} .
$$

Therefore, the $f_{\mathrm{N}}(T)$ and IS $(T)$ obtained from Mössbauer spectra will yield values 
of $\theta_{\mathrm{D}}$ by obtaining the slope of the logarithm of the first with respect to $T$ and by fitting the second with eq. (23). As only cores (particles) at rest contribute to the Mössbauer spectrum, IS $(T)$ refers to the correct $\theta_{\mathrm{D}}$, associated with lattice vibrations. When the cores (particles) are in motion, $f_{\mathrm{N}}(T)$ decreases more strongly with $T$ than predicted by (2) and hence $\theta_{\mathrm{D}}$ derived from the temperature dependence of the recoilless fraction will be too small. Hence, the greater the discrepancy between the $\theta_{\mathrm{d}}$ 's obtained from the $f_{\mathrm{N}}(T)$ and IS $(T)$ data, the greater the core (particle) motion contribution to the Mössbauer reaction $f_{N}(T)$. The $f$-factor (corrected spectral area) values (see fig. 6) of the $35 \mathrm{mg} \mathrm{PIC} / \mathrm{cm}^{2}$ absorber are used to calculate $\ln f_{\mathrm{N}}(T)$ for $T \geqslant 150 \mathrm{~K}$. Fig. 13 shows the plot of $\ln f_{\mathrm{N}}(T)$ versus $T$ for PIC. The slope of this figure and eq. (24) yield a value for the Debye temperature equal to $\theta_{\mathrm{D}}=248 \pm 12 \mathrm{~K}$. Fig. 14 shows the dependence of the isomer shifts: $(1-[\operatorname{IS}(0)-\operatorname{IS}(T)])$ of PIC on temperature, where we assume $\operatorname{IS}(T=4.2 \mathrm{~K})$ $=\mathrm{IS}(T=0 \mathrm{~K})$. The solid line represents a fit to the data using eq. (23) with a value of $\theta_{\mathrm{D}}=270 \mathrm{~K}$. However, one must note that values of $\theta_{\mathrm{D}}=250$ and $290 \mathrm{~K}$ in eq. (23) will produce curves passing through the lower and upper limits of the experimental error bars of the data. Hence, we can conclude that the fit of the PIC isomer shift data yields $\theta_{\mathrm{D}}=270 \pm 20 \mathrm{~K}$. The close agreement between the Debye temperatures obtained from the behavior of the Mössbauer fraction and the isomer shift data of PIC in the high temperature range 150-295 K, indicates that core motion is relatively weak and is not responsible for the anomaly. We also included in fig. 6 a fit to the data (the solid curve) obtained from the Debye model (eq. (22)) and $\theta_{D}=248 \mathrm{~K}$. We note that portions of the solid curve in the vicinity of the temperature range of the anomaly lie outside of the error bars.

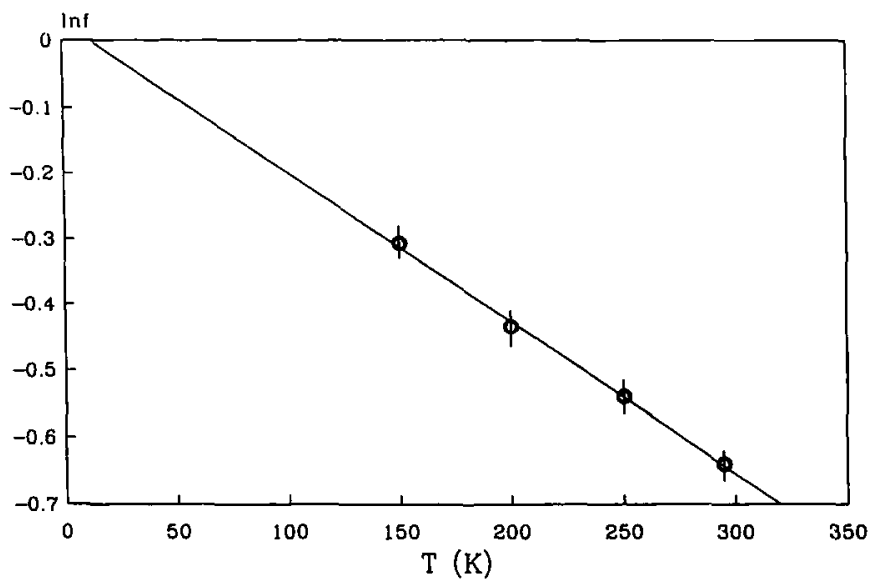

Fig. 13. $\ln f_{\text {Nexp }}(T)$ versus temperature, for the $35 \mathrm{mg} / \mathrm{cm}^{2}$ PIC absorber. The slope gives $\theta_{\mathrm{D}}=248 \pm 12 \mathrm{~K}$. 


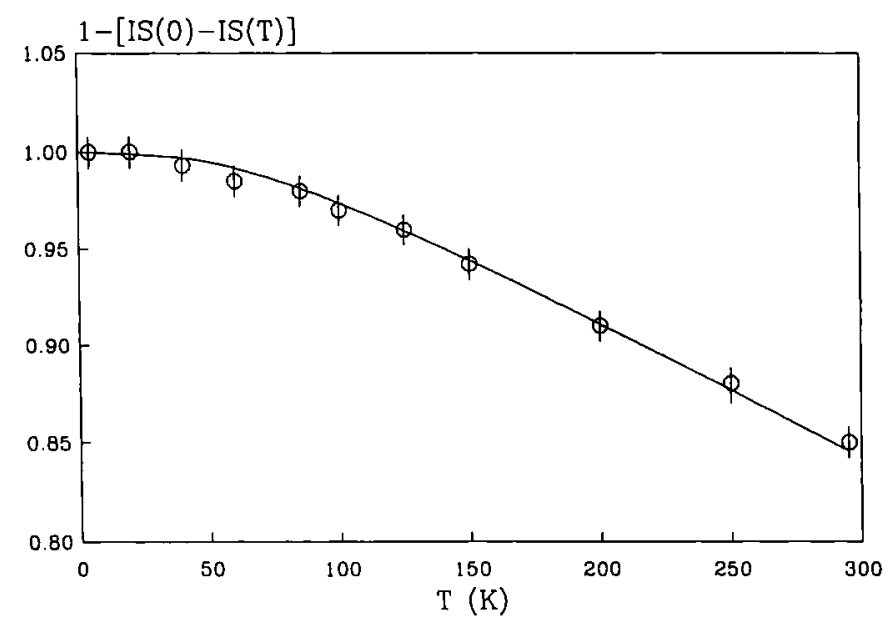

Fig. 14. The isomer shift $(1-[I S(0)-I S(T)])(\mathrm{mm} / \mathrm{s})$ as a function of temperature for the $10 \mathrm{mg} /$ $\mathrm{cm}^{2}$ PIC absorber (see table 1). The solid line represents the fit to the data with eq. (23).

\section{Conclusion}

Based on our results, we believe that the $f$-factor anomaly in ferritin and PIC is genuine and that neither absorber thickness effects nor motion of the core with respect to the protein shell is the main mechanism underlying this phenomenon. The experimental results indicate (section 5.2.1) that PIC and ferritin possess a magnetic anisotropy energy which is not strictly uniaxial. We have proposed [43-45] a mechanism involving magnetostriction as a theoretical basis of the anomaly, concisely: superparamagnetic relaxation brings about a dynamical displacement of the Mössbauer nucleus through magnetostriction. These displacements produce a Doppler broadening of the Mössbauer spectrum. Spectral area is lost in the background and results in a reduction of the apparent $f$-factor. Interestingly, the mechanism of magnetostriction broadening requires that the magnetic anisotropy energy not be uniaxial.

The possibility of using two Debye temperatures to fit the anomalous $f$-factor curve has been proposed [46]. This implies that the phonon frequency spectrum of ferritin and PIC are multi-peaked, which might account for the anomaly in the $f_{\mathrm{N}}(T)$. However, we have found that the inflection point of the anomalous region in the $f$-factor curve, for different samples, always occurs near their respective blocking temperatures. The relationship between the $f_{\mathrm{N}}(T)$ and the blocking temperature can be further investigated by varying the blocking temperature for different samples while keeping their Debye temperature(s) constant. This can be accomplished by applying a magnetic field to the samples, which would indicate whether the inflection point of the $f$-factor $f_{\mathrm{N}}(T)$ curve, being approximately at the blocking temperature is a coincidence or not. 
Further, it would be interesting to look for this anomaly in hemosiderin, other ferritin-like molecules [47], and more generally in other single domain magnetic particles whose magnetic anisotropy energy is not uniaxially symmetric.

\section{Acknowledgement}

We wish to thank Professor J. Teillet of The University of Rouen, Rouen, France for allowing us the use of his Mössbauer spectra fitting program. Also, thanks is due to Dr. W.F. Davis for discussions on FFT techniques. MEYME and GCP were partially supported by the Office of Naval Research under Grant no. N00014-89-J-1779. RBF was partially supported by the National Institute of Health under Grant no. 50R1 DK36799-05.

\section{References}

[1] J. Yariv et al., Biochem. J. 197 (1981) 171.

[2] E.I. Stiefel and G.D. Watt, Nature 279 (1979) 81.

[3] E.C. Theil, in: Advances in Inorganic Biochemistry, Vol. 5, eds. E.C. Theil, G.L. Eichorn and L.G. Marzilli (Elsevier, Amsterdam, 1983).

[4] K.M. Towe and W.F. Bradley, J. Colloid, Interface Sci. 24 (1967) 384.

[5] C.C. Ford et al., Phil. Trans. Roy. Soc. B 304 (1984) 551.

[6] D.W. Rice et al. in: Advances in Inorganic Biochemistry, Vol. 5, eds. E.C. Theil, G.L. Eichorn and L.G. Marzilli (Elsevier, Amsterdam, 1983);

P.M. Harrison, in: Advances in Inorganic Biochemistry, Vol. 5, eds. E.C. Theil, G.L. Eichorn and L.G. Marzilli (Elsevier, Amsterdam, 1983).

[7] D.M. Lawson et al., Nature 349 (1991) 542.

[8] D.M. Lawson et al., FEBS Lett. 254 (1989) 207.

[9] E.R. Bauminger et al., Biochem. Biophys. Acta 1118 (1991) 48.

[10] D.L. Jacobs et al., Biochemistry 28(1989) 1650.

[11] A. Treffy et al., FEBS Lett. 302 (1992) 108.

[12] A. Blaise et al., Compt. Rend. Acad. Sci. Paris 261 (1965) 2310; 265 (1967) 1077.

[13] R.B. Frankel et al., Hyp. Int. 33 (1987) 233.

[14] B. Kolk, in: Dynamical Properties of Solids, Vol. 5, eds. G.K. Horton and A.A. Maradudin (North-Holland, Amsterdam, 1984);

H. Frauenfelder, The Mössbauer Effect (Benjamin, New York, 1963).

[15] J.F. Sanders, Mich. Med. 7 (1968) 726;

R.W. Nexton et al., Clin. Trials J. 17 (1980) 106;

O.W. Crawford, Ill. Med. J. (1970).

[16] K.A. Berg et al., J. Inorg. Biochem. 22 (1984) 125.

[17] M-E.Y. Mohie-Eldin, PhD Thesis, Tufts University, Medford, MA, USA(1992).

[18] U.S. Patent No. 3821192 process for preparing an iron-saccharide complex.

[19] T.G. Spiro and P. Saltman, Struct. Bonding 6(1969) 116;

P.J. Murphy et al., J. Colloid Interf. Sci. 56(1976) 312.

[20] M-E.Y. Mohie-Eldin, R.B. Frankel and L. Gunther, J. Magn. Magn. Mater. 135(1994) 65.

[21] F. Varret, J. Phys. Chem. Solids 37 (1976) 265. 
[22] N.N. Greenwood and T.C. Gibb, Mössbauer Spectroscopy (Chapman and Hall, London, 1971).

[23] V.I. Goldanskii and R.H. Herber, Chemical Applications of Mössbauer Spectroscopy (Academic Press, New York, 1968).

[24] J. Herberle and S. Franco, Z. Naturforsch. 10 (1968) 1439.

[25] S. Margulies and J.R. Ehrman, Nucl. Instr. Meth. 12(1961) 131.

[26] S.L. Ruby and J.M. Hicks, Rev. Sci. Instr. 33 (1962) 27.

[27] M.C.D. Ure and P.A. Flinn, in: Mössbauer Effect Methodology, Vol. 7, ed. I.J. Gruverman (Plenum Press, New York, 1971) p. 245.

[28] R.B. Frankel et al., Hyp. Int. 66(1991) 71.

[29] P. Roggwiller and W. Kundig, Solid State Commun. 12(1973) 901.

[30] S. Mørup and H. Topsøe, Appl. Phys. 11 (1976) 63.

[31] S. Mørup et al., J. Phys. (Paris) 37 (1976) C6-287.

[32] S. Mørup, Hyp. Int. 60 (1990) 959;

S. Mørup, J. Magn. Magn. Mater. 37 (1983) 39.

[33] J.M. Williams et al., Phys. Med. Biol. 23 (1978) 835.

[34] M. Abramowitz and I.A. Stegun, eds., Handbook of Mathematical Functions (Dover, New York, 1972) pp. 297, 305, 319.

[35] T.G. St. Pierre et al., J. Magn. Mater. 69 (1987) 276.

[36] M.P.A. Viegers and J.M. Trooster, Phys. Rev. B 15 (1977) 72.

[37] I.P. Suzdalev et al., Sov. Phys. JETP24 (1968) 79.

[38] M. Hayashi et al., J. Phys. C13 (1980) 681.

[39] A.M. van der Kraan, Phys. Stat. Sol. 18a (1973) 215.

[40] J.W. Niemantsverdriet et al., Phys. Lett. 100A 8 (1984) 445.

[41] J. Heberle, Möss. Effect. Meth. 7(1971) 299.

[42] K.A. Hardy et al., Nucl. Instr. Meth. 86(1970) 171.

[43] M-E.Y. Mohie-Eldin and L. Gunther, in: Proc. Int. Workshop on Studies of Magnetic Properties of Fine Particles and Their Relevance to Materials Science, Rome, eds. J.L. Dormann and D. Fiorani (Elsevier, Amsterdam, 1992) p. 403.

[44] M-E.Y. Mohie-Eldin and L. Gunther, J. Magn. Magn. Mater. 127(1993) 346.

[45] L. Gunther and M.-E.Y. MOhie-Eldin, J. Magn. Magn. Mater. 129 (1994) 334.

[46] C. Hawkins, private communication.

[47] S.H. Bell, Biochem. Biophys. Acta. 787 (1984) 227;

E.R. Bauminger, Biochem. Biophys. Acta. 623 (1980) 237. 